\title{
VLT/NACO near-infrared imaging and spectroscopy of N88A in the SMC ${ }^{\star}$
}

\author{
G. Testor ${ }^{1, \star \star}$, J. L. Lemaire ${ }^{1,2, * *}$, M. Heydari-Malayeri ${ }^{1}$, L. E. Kristensen ${ }^{3}$, S. Diana ${ }^{2}$, and D. Field ${ }^{4}$ \\ 1 LERMA, UMR 8112 du CNRS, Observatoire de Paris, 92195 Meudon, France \\ e-mail: gerard.testor@obspm.fr \\ 2 Université de Cergy-Pontoise, 95031 Cergy Cedex, France \\ 3 Leiden Observatory, Leiden University, Niels Bohrweg 2, 2333 CA Leiden, The Netherlands \\ 4 Department of Physics and Astronomy, Århus University, 8000 Århus C, Denmark
}

Received 4 February 2009 / Accepted 24 November 2009

\section{ABSTRACT}

\begin{abstract}
Aims. We present near-infrared imaging and spectroscopic high spatial resolution observations of the SMC region N88 containing the bright, excited, extincted and compact $\mathrm{H}$ II region N88A of the size of about $1 \mathrm{pc}$.

Methods. To investigate its stellar content and reddening, N88 was observed using spectroscopy and imagery in the $J H K$ s- and $L^{\prime}$-band at a spatial resolution of $\sim 0.1-0.3^{\prime \prime}$, using the VLT UT4 equipped with the NAOS adaptive optics system. In an attempt to establish if the origin of the infra-red (IR) excess is due to bright nebulosity, circumstellar material and/or local dust, we used $K$ s vs. $J-K$ color-magnitude (CM) and $J H K$ color-color (CC) diagrams, as well as $L^{\prime}$ imagery.

Results. Our IR-data reveal in the N88 area an IR-excess fraction of $\geq 30$ per cent of the detected stars as well as an unprecedently detailed morphology of N88A. It consists of an embedded cluster of $\sim 3.5^{\prime \prime}(\sim 1 \mathrm{pc})$ in diameter of at least thirteen resolved stars superposed with an unusual bright continuum centered on a very bright star. The four brightest stars in this cluster lie red-ward of $H-K \geq 0.45$ mag and could be classified as young stellar object (YSO) candidates. Four other probable YSO candidates are also detected in $\mathrm{N} 88$ along a north-south bow-shaped thin $\mathrm{H}_{2}$ filament at $\sim 7^{\prime \prime}$ east of the young central bright star. This star, which we assume to be the main exciting source, could also be complex. At $0.2^{\prime \prime}$ east of this star, a heavily embedded core is detected in the $L^{\prime}$-band. This core with $L^{\prime} \sim 14 \mathrm{mag}$ and $L^{\prime}-K \geq 4.5 \mathrm{mag}$ could be a massive class I protostar candidate. The $2.12 \mu \mathrm{m} \mathrm{H}_{2} \mathrm{image}$ of N88A resembles a shell of a diameter of $\sim 3^{\prime \prime}(\sim 0.9 \mathrm{pc})$ centered on the bright star. This shell consists of three bright components, of which the brightest one superposes the ionization front. The line ratios of $\mathrm{H}_{2} 2-1 \mathrm{~S}(1)$ and 1-0 $\mathrm{S}(0)$ relative to 1-0 S(1), as well as the presence of high $v$ lines, are indicative of photodissociation regions, rather than shocks.
\end{abstract}

Key words. stars: formation - circumstellar matter - ISM: individual: N88A - HII regions - dust, extinction Magellanic Clouds

\section{Introduction}

The Small Magellanic Cloud (SMC) is rich in H II regions and young $\mathrm{OB}$ associations. Because of its known and relatively small distance ( $\sim 65 \mathrm{kpc}$ ) (Kovacs 2000$)$, its face-on position relatively free from foreground extinction (McNamara \& Feltz 1980), and low internal extinction (Westerlund 1997), it is well-suited for the study of both individual stars and very compact objects as well as global structures. It is an ideal laboratory for studying the formation and evolution of massive stars in a low metallicity environment. Understanding the characteristics of massive stars and their interaction with their environment is a key problem in astrophysics. We have made some progress concerning the early stages of massive star formation in the galaxy, but the current knowledge about the early stages of massive star evolution in other galaxies is mediocre at best. The main reason is that the earliest stages of massive star evolution are deeply enshrouded, inaccessible in the optical wavelengths. Another reason is that these stars are often members of very crowded regions. Today, high-spatial near-infra-red (NIR) resolution observations using NACO attached to the Very

* Based on observations obtained at the European Southern Observatory, El Paranal, Chile

$\star \star$ Visiting astronomer at VLT Paranal.
Large Telescope (VLT) are able to overcome these obstacles in the SMC. Our search for the youngest massive stars in the Magellanic Clouds (MCs) (Heydari-Malayeri \& Testor 1982) led to the discovery of a distinct and very rare class of $\mathrm{H}$ II regions that we called high-excitation compact H II "blobs" (hereafter HEBs) listed in Testor (2001). In contrast to the ordinary $\mathrm{H}$ II regions of the MCs, which are extended structures spanning several arcminutes on the sky $(>50 \mathrm{pc})$ and are powered by a large number of hot stars, HEBs are very dense small regions, usually $2^{\prime \prime}$ to $8^{\prime \prime}$ in diameter ( 0.8 to $3 \mathrm{pc}$ ), ionized by one or a few massive stars and affected by local dust. Two other HII regions, MA 1796 and MG 2 (less than 1 pc across), which are heavily extincted and ionized by a small young cluster, have been found by Stanghellini et al. (2003) in the SMC.

In the present paper we focus on the peculiar HEB LHA 115-N88A, hereafter labelled N88A, which is of a diameter of $\sim 1$ pc (Testor \& Pakull 1985) in the extended H II region LHA 115-N88 or N88 (Henize 1956), which in turn is of a diameter of $\sim 10 \mathrm{pc}$. N88 lies in the Shapley Wing of the SMC and contains the young cluster HW $81\left(\sim 0.6^{\prime}\right)$ (Hodge \& Wright 1977). It is known that the SMC is made of four H I layers with different velocities (McGee \& Newton 1982). This situation complicates the study, particularly in the region of the Shapley wing. However, the available H I observations provide 
helpful data for the study of this region. In particular, the N88 region lying at about $35^{\prime}(\sim 700 \mathrm{pc})$ west of N83/N84 is apparently not associated with the $\mathrm{H}$ I cloud of these H II regions (Heydari-Malayeri et al. 1988). N88A should be associated with the H I component of the velocity of $134 \mathrm{~km} \mathrm{~s}^{-1}$ (McGee \& Newton 1982).

N88A is the brightest and the most excited HEB in the MCs. It is also the most reddened $\mathrm{H}$ II region in these galaxies of lowmetallicity (Heydari-Malayeri et al. 2007). Numerous optical detailed studies have been made on this object (Testor \& Pakull 1985; Wilcot 1994; Heydari-Malayeri et al. 1999, hereafter HM99; Kurt et al. 1999; Testor et al. 2003). Nevertheless, many uncertainties remain to understand the true nature of N88A, such as its exciting source, which still remains unidentified, and also the nature of the reddening.

Israel \& Koorneef $(1988,1991)$ have detected the presence of molecular hydrogen in N88 through $\mathrm{H}_{2}$ emission which is either shock-excited on a small scale of $0.46^{\prime \prime}$ by stars embedded in the molecular cloud, or radiatively excited on a large scale $\left(3^{\prime \prime}-60^{\prime \prime}\right)$. But their low spectral $(R=50)$ and spatial (7.5-10" aperture) resolutions did not allow them to distinguish between these different processes. They described N88A as a strong NIR source dominated by nebular emission containing a strong hot dust component and noticed that N88A has an unusual blue $J-H$ color. In Testor et al. (2005) a pure $\mathrm{H}_{2}$ emission is detected in N88A at low spatial resolution, as well as along a north-south diffuse long filament at $\sim 6-8^{\prime \prime}$ to the east. In N66 (Henize 1956), which is a giant HII region in the SMC, Schmeja et al. (2009) have reported that most of the $\mathrm{H}_{2}$ emission peaks coincide with the bright component of the ionized gas and with compact embedded young clusters where candidate YSOs have been identified. Using SEST, Israel et al. (2003) detected a $\mathrm{CO}$ molecular cloud of $1.5^{\prime} \times 1.5^{\prime}$ in the region, reporting spectra and maps of the ${ }^{12} \mathrm{CO}$ lines $J=1-0$ and $J=$ 2-1. Stanimirovic et al. (2000) found that the highest values of the dust-to-gas mass ratio and dust temperature in the SMC are found in N88A.

IR studies of a similar size young star formation region like the Trapezium region in Orion $(\sim 0.75 \times 0.75 \mathrm{pc})$ (Lada et al. 2000) and the more extended 30 Doradus in the LMC (Maercker \& Burton 2005) showed that during star formation, YSOs are associated with the circumstellar material inducing IR-excess emission, and also that the use of $J H K$ CC diagrams are useful tools to detect this emission. However, for young massive stars generally found in embedded clusters, their surrounding material destruction time scale is short, which renders their observation difficult (Bik et al. 2005, 2006). The most suitable wavelength to determine the nature of the IR-excess is the $L$-band, increasing the IR-excess and reducing the contribution of extended emission from reflection nebulae and H II regions (Lada et al. 2000). IR-excess can be useful to determine the origin of the reddening in embedded young clusters. Martin-Hernandez et al. (2008) found in N88A a rising dust continuum and PAH bands from a mid-IR high spatial resolution Spitzer-IRS spectrum typical characteristics in $\mathrm{H}$ II regions. Using radio observations, Indebetouw et al. (2004) found that N88A is ionized by an O5 type star.

In the present paper we present the results of $J H K$ - and $L^{\prime}$-band high spatial resolution observations of N88A and its surroundings, using adaptive optics at the VLT. Section 2 discusses the instrumentation employed during these observations and the data acquisition and reduction procedures used. Section 3 describes the results and analysis of our observations, and Sect. 4 gives our conclusions.

\section{Observations and data reduction}

NIR observations of N88A were obtained at the ESO VLT during October 2004. Images and spectra were taken using NACO on UT4, composed of the Nasmyth Adaptive Optics System (NAOS) and the High Resolution IR Camera and Spectrometer (CONICA). The detector was a $1026 \times 1024$ SBRC InSb Alladin 3 array. The cameras S54 and S27 were used in the range of $1.0-2.5 \mu \mathrm{m}$ and the $\mathrm{L} 27$ camera in the range of $2.5-5.0 \mu \mathrm{m}$.

The field-of-view (FOV) of the S54 camera was 54" $\times 54^{\prime \prime}$ with a pixel size of $0.05274^{\prime \prime}$, corresponding to $0.015 \mathrm{pc}$ at the distance modulus of 19.05 for the SMC (Kovacs 2000). The FOV of the S27 and L27 camera was $27.15^{\prime \prime} \times 27.15^{\prime \prime}$ with a pixel size of $0.02637^{\prime \prime}$, corresponding to 0.0075 pc. For spectroscopy we used the S54 camera.

As an adaptive optics (AO) reference source for wavefront sensing we used the object itself (N88A) (Testor et al. 2005). The conditions were photometric, and the seeing ranged from $0.65^{\prime \prime}$ to $1^{\prime \prime}$ in the visible. After subtraction of the average dark frame, each image was divided by the normalized flat field image. The data were reduced mainly with the ESO software packages MIDAS and ECLIPSE.

\subsection{Imaging}

Images through $J, H, K$ s broad-band and the $2.12 \mu \mathrm{m}, 2.24 \mu \mathrm{m}$ narrow band filters were obtained with the S54 camera. A composite $J H K$ s color image of the observed field is shown in Fig. 1. Images with higher spatial resolution in the $K$ s and $L^{\prime}$ large band were also obtained with the S27 camera. The log of the NIR imaging observations is given in Table 1 . The AutoJitter mode was used: that is, the telescope moves at each exposure according to a random pattern in a $6^{\prime \prime} \times 6^{\prime \prime}$ box. Table 1 lists the stellar full-width-at-half-maximum $(F W H M)$ in final images of different observed bands of the star at J2000 coordinates $(\alpha, \delta)=$ $\left(1^{\mathrm{h}} 24^{\mathrm{m}} 8.88^{\mathrm{s}},-73^{\circ} 8^{\prime} 56.2^{\prime \prime}\right)$ of the 2MASS survey (Cutri et al. 2003). The AO image is affected by anisoplanatism and leads to a degradation of the point spread function (PSF) becoming more elongated as the angular offset from the guide star increases. This has been taken into consideration for the photometric measurements, as explained in Sect. 2.3.

\subsection{Spectroscopy}

Spectroscopy was performed in the S54-4-SHK mode (broadband filter), giving a linear dispersion of $1.94 \mathrm{~nm} / \mathrm{pixel}$ and a spatial scale of 53 mas/pixel. Three long-slit spectra S1-S3 were chosen from the IR images given by NACO. S1 $\left(\mathrm{PA}=115^{\circ}\right)$ and S2 $\left(\mathrm{PA}=89.3^{\circ}\right)$ cross the central bright star. S3 $\left(\mathrm{PA}=18.9^{\circ}\right)$ crosses the stars \#37 and \#47 (see Fig. 3). The slit width was 172 mas and the spectral resolution $\sim 400$. For each exposure the detector integration time (DIT) was $100 \mathrm{~s}$. Ten exposures were obtained in the Autonod on slit mode, which allows the spectroscopy of moderately extended objects. The log of spectroscopic observations is given in Table 2. In order to remove telluric absorption features, stars with a similar airmass were observed as telluric standards. Spectroscopy was reduced with the MIDAS software package LONG.

\subsection{Photometry}

In Fig. 2 we present the N88 region observed with the S54 camera (Field 1) through the $J$-band filter. The instrumental magnitudes of the elongated stars (see Sect. 2.1) outside the central 


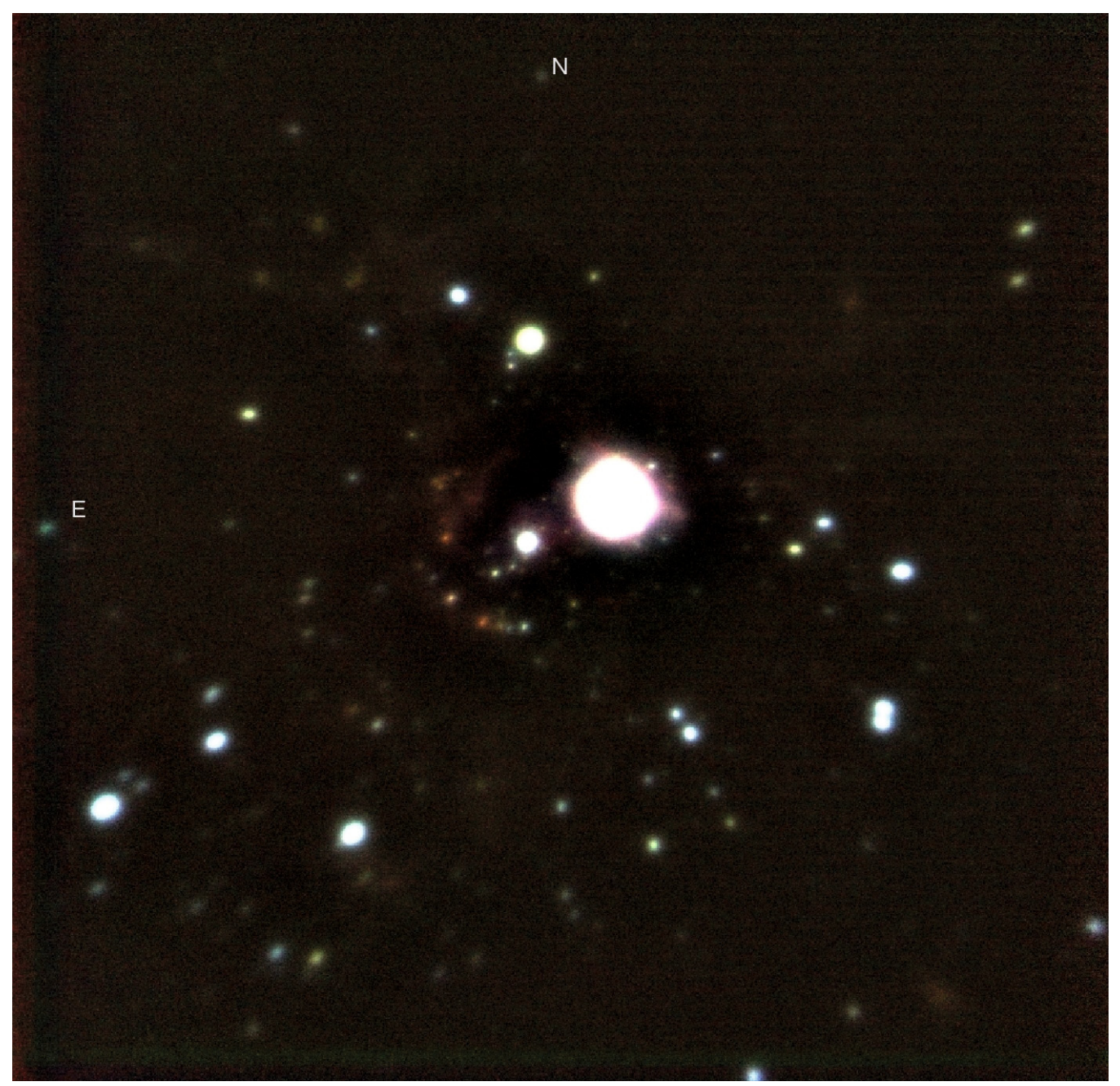

Fig. 1. JHK color-composite image of SMC N88 containing the bright HEB N88A. The image size extracted from the S54 camera field corresponds to $50^{\prime \prime} \times 50^{\prime \prime}$ (or $\sim 14 \mathrm{pc} \times 14 \mathrm{pc}$ ). The lower spatial density of faint stars close to the edges of the frame delimits approximatly the cluster N88-cl. In the north-west quadrant the lack of stars is explained by the presence of the molecular cloud (HM99). A red filament is visible east of N88A. $J$, $H$ and $K$ are respectively coded $\mathrm{B}, \mathrm{G}$ and $\mathrm{R}$. North is up and East is left.

Table 1. Log of the photometric VLT/NACO observations.

\begin{tabular}{|c|c|c|c|c|c|c|}
\hline Id. & Filter & $\begin{array}{l}\text { Expo. } \\
t(\mathrm{~s}) \times n\end{array}$ & Mode & Date & $\begin{array}{c}\text { Seeing } \\
\left({ }^{\prime \prime}\right)\end{array}$ & $\begin{array}{c}F W H M \\
\left({ }^{\prime \prime}\right)\end{array}$ \\
\hline \multirow[t]{7}{*}{$\overline{\mathrm{N} 88 \mathrm{~A}}$} & $J 1.27 \mu \mathrm{m}$ & $20 \times 30$ & - & - & - & 0.35 \\
\hline & $H 1.66 \mu \mathrm{m}$ & $20 \times 30$ & - & - & & 0.27 \\
\hline & $K \mathrm{~s} 2.18 \mu \mathrm{m}$ & $30 \times 30$ & S54 & $2004-10-9$ & $0.6-0.9$ & 0.21 \\
\hline & $L^{\prime} 3.8 \mu \mathrm{m}$ & $0.2 \times 26$ & S27 & $2004-10-10$ & $0.9-1.2$ & 0.10 \\
\hline & NB-2.12 & $200 \times 20$ & S54 & - & & 0.19 \\
\hline & NB-2.24 & $150 \times 20$ & S54 & - & & 0.25 \\
\hline & $K \mathrm{~s}$ & $60 \times 30$ & S27 & 2004-10-11 & & 0.10 \\
\hline
\end{tabular}

region were derived with DAOPHOT (Stetson 1987), using concentric aperture photometry to integrate all the flux of each star. Although PSF photometry is better adapted for crowded fields, we could not use it. Indeed the stars in our field were too faint and/or crowded to obtain the number of PSF stars necessary to use the photometric analysis elaborated by Pugliese et al. (2002), taking into account the $\mathrm{AO}$ anisoplanatism effect.

The detected stars are identified by a number referring to Col. 1 of Table 3 that gives the astrometry and photometry. The
Table 2. Log of the VLT/NACO long-slit spectroscopic observations.

\begin{tabular}{llccccc}
\hline \hline Id. & Date & $\begin{array}{c}\text { Slit } \\
(\mathrm{mas})\end{array}$ & $\begin{array}{c}\text { Exposures } \\
t(\mathrm{~s}) \times n\end{array}$ & Mode & $\begin{array}{c}\lambda / \delta \lambda \\
\begin{array}{c}\text { Seeing } \\
\left({ }^{\prime \prime}\right)\end{array}\end{array}$ \\
\hline S1 & $2004-10-9$ & 172 & $100 \times 10$ & S54-4-SHK & 400 & $0.6-0.9$ \\
S2 & $2004-10-10$ & - & - & - & - & - \\
S3 & $2004-10-11$ & - & - & - & - & - \\
& & & & & & \\
hip8485 & $2004-10-9$ & - & $2.5 \times 4$ & - & - & - \\
hip29968 & $2004-10-9$ & - & $2 \times 4$ & - & - & - \\
hip103087 & $2004-10-11$ & - & $1.8 \times 4$ & - & - & - \\
\hline
\end{tabular}

central object N88A is not affected by anisoplanatism, so the $J H K$ instrumental magnitudes of the stars were derived using the DAOPHOT's multiple-simultaneous-profile-fitting photometry routine (NSTAR), which is well adapted for photometry in crowded fields. The detected stars are shown in the inset of Fig. 2 and are also listed in Table 3. Almost all the stars of Field 1 (Fig. 2) have photometric uncertainties in the $J$-, $H$ - and $K$-band, less than $0.03 \mathrm{mag}$ for stars with $K<16 \mathrm{mag}$, less than 0.06 


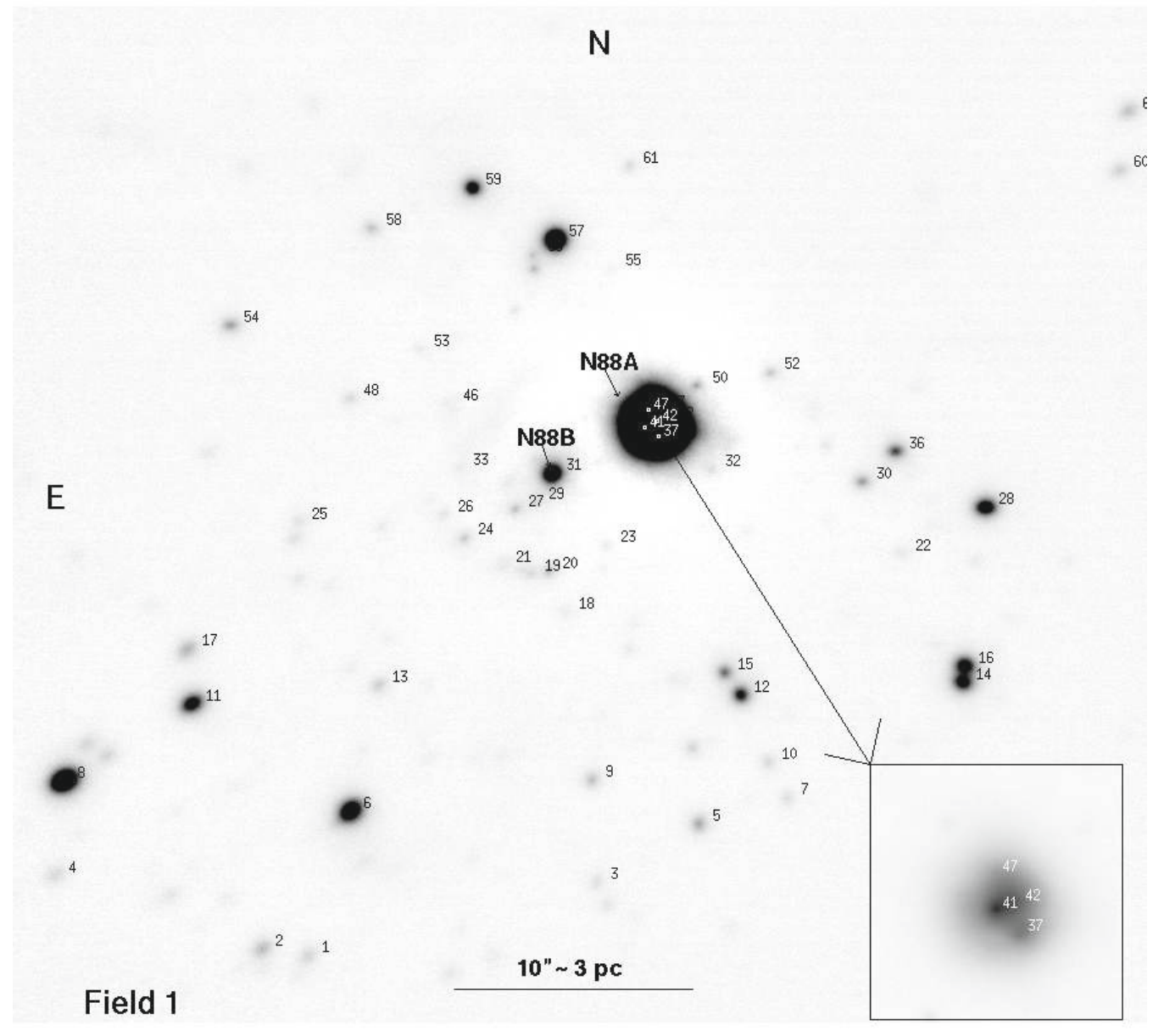

Fig. 2. Finding chart in the $J$-band obtained with the S54 camera (Field 1). The inset corresponds to an enlarged (factor two) and unsaturated image of N88A extracted from Field 1. The numbering refers to Table 3. The total field size corresponds to $48.6^{\prime \prime} \times 47.5^{\prime \prime}($ or $\sim 14 \mathrm{pc} \times 14 \mathrm{pc}$ ).

for stars with $16<K<18$ mag and greater than 0.1 for stars $18<K<20$ mag.

Figure 3 (Field 2) shows only a part of N88 observed with the S27 camera through the $K$ s-band filter. On this figure the directions of the spectra $S 1-S 3$ are plotted. This camera, with a pixel two times smaller than S54, has a better spatial resolution, so the analysis of the crowded field of N88A with the routine NSTAR which allows us a more accurate star detection. In Fig. 4a the stars of N88A are identified by a number referring to Col. 1 of Table 4. In this table, the average photometric errors of the stars reported by DAOPHOT are $\sim 0.04$ mag in $J$ and 0.06 in $H$ and $K$ s for stars of magnitude $\leq 16$ except for the bright isolated star \#57 outside N88A ( 0.03 and $\sim 0.05 \mathrm{mag})$. This star will be used as PSF. The photometric calibration was obtained using the isolated 2MASS star at J2000 $(\alpha, \delta)=\left(1^{\mathrm{h}} 24^{\mathrm{m}} 8.88^{\mathrm{s}},-73^{\circ} 8^{\prime} 56.2^{\prime \prime}\right)$ corresponding to our star \#57. The conversion of pixel coordinates to $\alpha$ and $\delta$ was derived using the same star, and the relative positions of our stars are accurate to better than $0.1^{\prime \prime}$.

In the core of N88A the determination of the sky aperture parameters used in NSTAR is very sensitive, even with the S27 camera. Indeed, the wings of the stars superpose the wings of the strong continuum. The distribution of this continuum resembles a Gaussian profile (Fig. 5). The error on the magnitude of these stars, due to a steeply sloping continuum background, is greater than the error given by DAOPHOT. Because of this situation, the $K$ magnitude of the central star labelled \#41 at low and at higher spatial resolution (Field 1 and Field 2) is respectively 13.82 and $14.99 \mathrm{mag}$. Therefore, the $K$ magnitudes of the stars \#37, \#41, and \#47 (Field 2) were remeasured by subtracting a one-dimensional (1D) profile corresponding to the PSF crossing the center of the isolated reference star \#57. The magnitude of the PSF was multiplied by a factor in a way that only the continuum remains visible. In this case its magnitude corresponds to the magnitude of the star. An example is given in Fig. 5. The $K$ magnitudes of the stars obtained with this method are listed in Col. 7 of Table 4 . In this table the magnitude of \#41 agrees with the magnitude obtained with NSTAR (Col. 5), while for \#37, \#42 and \#47 the Ks magnitude is greater. Several faint stars below the detection level or slightly extended are not detected or rejected by DAOPHOT (Fig. 4b). In our underexposed $L^{\prime}$-band frame of N88, not shown here, contrary to the $J H K$-band no star is found apart from star \#57. However, in N88A the bright star \#41 is visible as well as the stars \#37, \#42, $\# 47$ and several unresolved features. A peculiar bright core labelled L1-C (see Sect. 3 below) located 0.2" east of \#41 is also found. This core coincides approximately with the HST absorption lane (HM99) and has a very faint counterpart in the $K$ s-band (Fig. 6). In order to derive the $L^{\prime}$ magnitude of these objects, we referred to the $L^{\prime}$ photometry of Israel \& Koorneef (1991), which was obtained through a $7 "$ aperture (Table 5). The integration of N88A in our sky subtracted $K$ s image, using a $4^{\prime \prime}$ aperture, gives a magnitude of 11.08, in agreement with Israel \& Koorneef (1991) and other authors (Table 5). In an aperture of 4" we have integrated the $L^{\prime}$ flux of N88A that was then calibrated with 
G. Testor et al.: NIR VLT/NACO observations of N88A in the SMC

Table 3. Stars in the region of N88A observed with the S54 camera (Field 1).

\begin{tabular}{|c|c|c|c|c|c|c|c|c|}
\hline Id. & $\alpha(2000)$ & $\delta(2000)$ & mag- $J$ & mag- $H$ & mag- $K$ & $J-K$ & $J-H$ & $H-K$ \\
\hline 1 & 12411.42 & 73926.15 & 18.24 & 17.65 & 17.50 & 0.74 & 0.59 & 0.15 \\
\hline 2 & 12411.86 & 73925.85 & 17.63 & 17.71 & 17.65 & -0.02 & -0.08 & 0.06 \\
\hline 3 & 1248.67 & 73923.08 & 19.08 & 18.88 & 18.84 & 0.24 & 0.20 & 0.04 \\
\hline 4 & 12413.84 & 73922.77 & 18.46 & 18.48 & 18.45 & 0.01 & -0.02 & 0.03 \\
\hline 5 & 1247.69 & 73920.63 & 17.89 & 17.61 & 17.34 & 0.55 & 0.28 & 0.27 \\
\hline 6 & 12411.02 & 73920.11 & 15.76 & 15.85 & 15.82 & -0.06 & -0.09 & 0.03 \\
\hline 7 & 1246.85 & 73919.56 & 19.16 & 18.71 & 18.51 & 0.66 & 0.45 & 0.21 \\
\hline 8 & 12413.75 & 73918.85 & 15.17 & 15.37 & 15.38 & -0.21 & -0.20 & -0.01 \\
\hline 9 & 1248.72 & 73918.79 & 18.22 & 18.18 & 18.02 & 0.20 & 0.04 & 0.16 \\
\hline 10 & 1247.03 & 73918.05 & 18.80 & 18.79 & 18.71 & 0.09 & 0.01 & 0.08 \\
\hline 11 & 12412.53 & 73915.61 & 16.27 & 16.45 & 16.47 & -0.20 & -0.18 & -0.02 \\
\hline 12 & 1247.30 & 73915.25 & 16.72 & 16.87 & 16.87 & -0.16 & -0.15 & -0.00 \\
\hline 13 & 12410.75 & 73914.84 & 18.67 & 18.53 & 18.06 & 0.61 & 0.14 & 0.47 \\
\hline 14 & 1245.18 & 73914.70 & 16.27 & 16.44 & 16.31 & -0.04 & -0.17 & 0.13 \\
\hline 15 & 1247.45 & 73914.32 & 17.31 & 17.48 & 17.47 & -0.15 & -0.17 & 0.01 \\
\hline 16 & 1245.16 & 73914.07 & 16.19 & 16.40 & 16.26 & -0.07 & -0.21 & 0.14 \\
\hline 17 & 12412.57 & 73913.33 & 17.44 & 17.59 & 17.40 & 0.04 & -0.15 & 0.19 \\
\hline 18 & 1248.97 & 73911.82 & 19.78 & 19.65 & 19.52 & 0.26 & 0.12 & 0.14 \\
\hline 19 & 1249.30 & 73910.17 & 18.44 & 18.21 & 17.87 & 0.57 & 0.23 & 0.34 \\
\hline 20 & 1249.13 & 73910.09 & 18.30 & 18.23 & 18.19 & 0.11 & 0.08 & 0.03 \\
\hline 21 & 1249.58 & 7399.84 & 19.11 & 18.51 & 17.66 & 1.45 & 0.60 & 0.84 \\
\hline 22 & 1245.76 & 7399.34 & 18.89 & 18.59 & 17.93 & 0.96 & 0.30 & 0.66 \\
\hline 23 & 1248.58 & 7399.01 & 19.65 & 19.34 & 18.95 & 0.70 & 0.31 & 0.39 \\
\hline 24 & 1249.93 & 7398.68 & 18.66 & 18.36 & 17.80 & 0.86 & 0.31 & 0.55 \\
\hline 25 & 12411.52 & 7398.03 & 18.89 & 18.73 & 18.51 & 0.38 & 0.15 & 0.22 \\
\hline 26 & 12410.13 & 7397.70 & 19.48 & 19.46 & 19.30 & 0.17 & 0.02 & 0.15 \\
\hline 27 & 1249.45 & 7397.50 & 18.37 & 18.18 & 17.97 & 0.40 & 0.19 & 0.20 \\
\hline 28 & 1244.96 & 7397.42 & 16.32 & 16.48 & 16.42 & -0.10 & -0.16 & 0.06 \\
\hline 29 & 1249.25 & 7397.17 & 18.21 & 18.11 & 17.88 & 0.33 & 0.10 & 0.24 \\
\hline 30 & 1246.14 & 7396.35 & 17.85 & 17.51 & 17.33 & 0.52 & 0.34 & 0.18 \\
\hline 31 & 1249.09 & 7395.99 & 15.74 & 15.73 & 15.51 & 0.23 & 0.01 & 0.16 \\
\hline 32 & 1247.57 & 7395.80 & 19.04 & 19.09 & 18.91 & 0.13 & -0.05 & 0.18 \\
\hline 33 & 1249.98 & 7395.77 & 19.46 & 18.76 & 17.83 & 1.63 & 0.70 & 0.93 \\
\hline 36 & 1245.82 & 7395.09 & 17.36 & 17.43 & 17.26 & 0.10 & -0.07 & 0.17 \\
\hline $37^{n}$ & 1247.84 & 7394.24 & 15.01 & 15.10 & 14.28 & 0.73 & -0.09 & 0.81 \\
\hline $41^{n}$ & 1247.96 & 7393.74 & 14.68 & 14.56 & 13.82 & 0.86 & 0.12 & 0.74 \\
\hline $42^{n}$ & 1247.85 & 7393.60 & 15.05 & 15.05 & 14.44 & 0.61 & 0.00 & 0.61 \\
\hline $47^{n}$ & 1247.96 & 7393.00 & 15.55 & 15.36 & 14.88 & 0.67 & 0.19 & 0.48 \\
\hline 46 & 12410.08 & 7393.05 & 19.65 & 18.93 & 18.18 & 1.47 & 0.72 & 0.75 \\
\hline 48 & 12411.02 & 7392.86 & 18.93 & 18.90 & 18.82 & 0.12 & 0.03 & 0.09 \\
\hline $50^{n}$ & 1247.53 & 7392.09 & 17.61 & 17.62 & 17.16 & 0.45 & -0.01 & 0.46 \\
\hline 52 & 1247.01 & 7391.79 & 18.80 & 18.98 & 18.57 & 0.23 & -0.18 & 0.41 \\
\hline 53 & 12410.35 & 7390.80 & 19.91 & 19.51 & 18.95 & 0.96 & 0.40 & 0.56 \\
\hline 54 & 12412.17 & 73859.81 & 17.75 & 17.59 & 17.29 & 0.46 & 0.16 & 0.30 \\
\hline 55 & 1248.53 & 73857.45 & 20.05 & 20.01 & 19.65 & 0.40 & 0.04 & 0.36 \\
\hline 56 & 1249.27 & 73856.87 & 17.57 & 17.42 & 17.29 & 0.28 & 0.15 & 0.13 \\
\hline 57 & 1249.07 & 73856.24 & 15.36 & 14.88 & 14.59 & 0.77 & 0.48 & 0.29 \\
\hline 58 & 12410.81 & 73855.78 & 18.45 & 18.43 & 18.26 & 0.19 & 0.02 & 0.17 \\
\hline 59 & 1249.86 & 73854.07 & 16.51 & 16.67 & 16.64 & -0.13 & -0.16 & 0.03 \\
\hline 60 & 1243.68 & 73853.33 & 18.51 & 18.03 & 17.59 & 0.92 & 0.48 & 0.44 \\
\hline 61 & 1248.36 & 73853.17 & 19.27 & 18.65 & 18.44 & 0.83 & 0.62 & 0.21 \\
\hline 62 & 1243.59 & 73850.89 & 18.06 & 17.65 & 17.43 & 0.63 & 0.41 & 0.22 \\
\hline
\end{tabular}

Notes. ${ }^{(n)}$ Stars in N88A analyzed using the NSTAR routine.

$L^{\prime}=8.92$ mag given by Israel \& Koorneef (1991). The $L^{\prime}$ magnitudes of the stars \#57, \#41 and the core L1-C (continuum subtracted) were obtained using an aperture of $0.35^{\prime \prime}$ and are listed in Table 4.

\section{Results and discussion}

\subsection{The HEB N88A}

The HST H $\alpha(\mathrm{F} 656 \mathrm{~N})$ and continuum Stromgren $y$ (F547M) images of N88A described in HM99 show two inhomogeneous wings separated by a north-south absorption lane (Fig. 4c). The western wing is much brighter and contains two faint stars, $\# 1$ and \#2, corresponding to our stars \#41 and \#42, as well as a "dark spot" to the south at the location of our star \#37. Figure 7 shows the intensity distribution plots in the Stromgren $y$ - and $K$ s-band in the direction of the slit S1 (Fig. 3), crossing N88B and the star \#41. In Fig. 7 the stars \#41 and \#42 of the magnitude of 14.99 and 16.11 respectively (Table 4) coincide with the two faint stars \#1 and \#2 (Fig. 4c) of $y=18.2 \mathrm{mag}$ and 18.3 mag (HM99). 


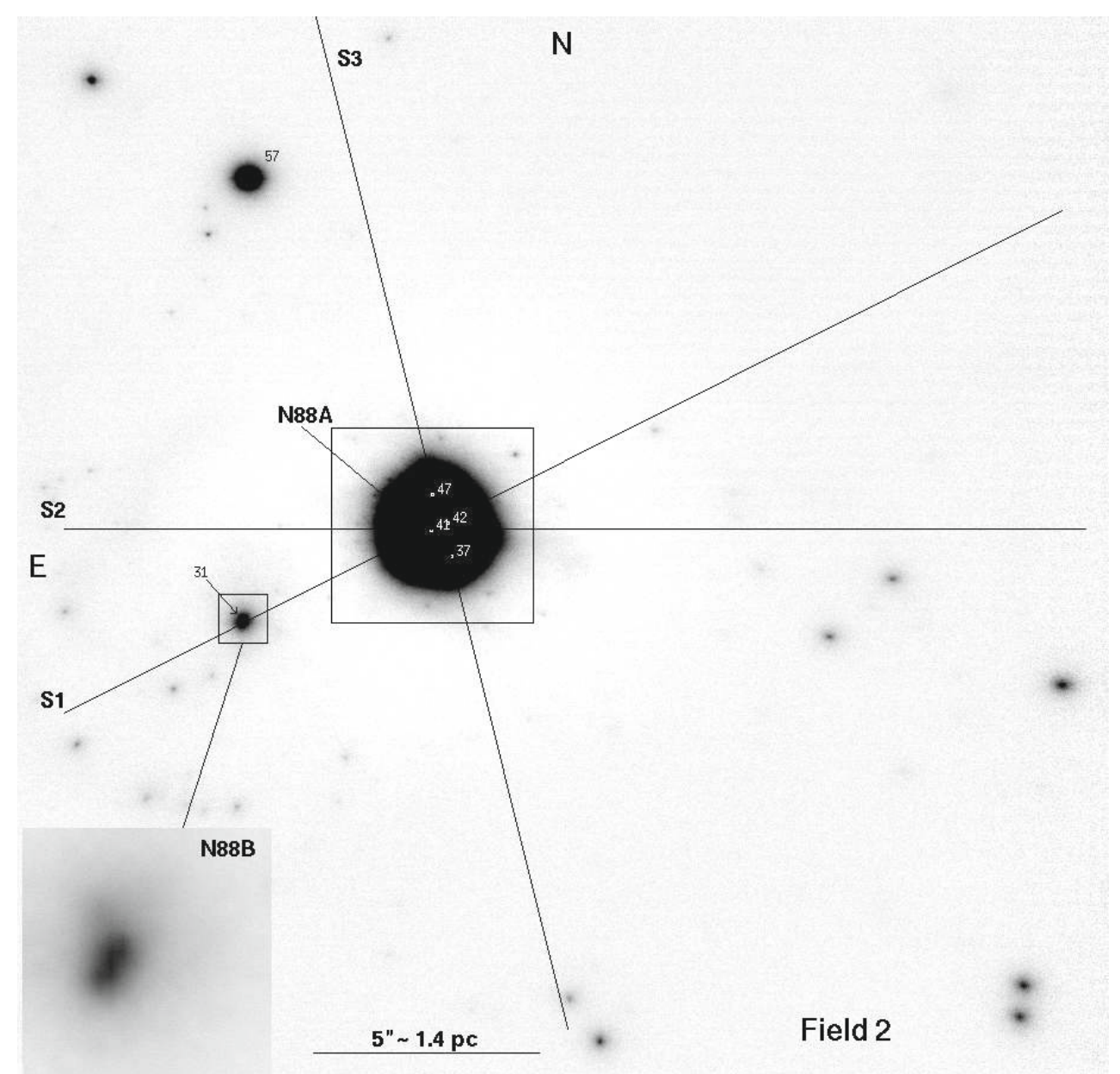

Fig. 3. Ks-band image of SMC-N88 obtained with the S27 camera (Field 2). The location of the slits labelled S1-S3 used in the spectroscopic mode is indicated by a solid line. In N88A the positions of the stars \#37, \#41, \#42 and \#47 are indicated. The bottom left is an enlargement of $0.55^{\prime \prime} \times 0.55^{\prime \prime}$ containing the complex object N88B. The image size corresponds to $25^{\prime \prime} \times 25^{\prime \prime}$ (or $\sim 7 \mathrm{pc} \times 7 \mathrm{pc}$ ).

Through the $K$ s filter (Figs. 4a and b), N88A appears as a circular nebular region of $\sim 3.4^{\prime \prime}$ diameter, centered on the relatively bright star \#41. This star coincides with the 2MASS point source 012407.92-730904.1 of $K=11.18 \mathrm{mag}$ (Cutri et al. 2003). In a diameter of $\sim 3.6^{\prime \prime}$ our N88A image exhibits a small embedded cluster labelled N88A-cl of at least thirteen stars (Fig. 4b). In Fig. 4b the usual Digital Development Process (DDP) introduced by K. Okano ${ }^{1}$ was applied to enhance the faint stars by compressing the range of brightnesses between the bright and dim portions of the image. The $K$ photometry of these stars is listed in Table 4. These stars mainly concentered to the north and east, superpose numerous nebular structures. It is interesting to see that these stars are aligned in the direction of the interface between the HII regions N88A and N88B (HM99).Through the $L^{\prime}$-band, N88A seems to be essentially formed by four distinct components labelled L1-L4 (Fig. 4d). L1, that contains the core labelled L1-C (Fig. 7), is the brightest and most compact component. In the Ks-band L1-C (Figs. 4a and 6) shows a very faint counterpart $(L-K \mathrm{~s} \geq 4.2)$. L2 and L3 are more diffuse and coincide with the stars \#47 and \#37. L4 is bright, extended and formed by two east-west elongated subcomponents spanning between stars \#47 and \#41. In the $L^{\prime}$-band the star \#41 is relatively bright $\left(L^{\prime} \sim 14.1 \mathrm{mag}\right)$ and well resolved (Fig. 4d). All these components superpose a diffuse nebular continuum. On the $y$ (F547M) continuum image (Fig. 4c) the "dark

${ }^{1}$ http://www .asahi-net.or.jp/ rt6k-okn/its98/ddp1.htm spot" corresponding to our component L3 is very bright. Neither the $y$ continuum structures located between stars \#41 and \#37, nor the one north of the "dark spot" (Fig. 4c) are seen in the $L^{\prime}$-band (Fig. 4d).

\subsection{The N88B region}

At the optical wavelengths, HM99 found that the central star of N88B, which corresponds to our star \#31, has an integrated magnitude of $y=16.57$ and consists of at least three components. Our high spatial resolution $K$ s-band image also shows that star \#31 of the magnitude of $K=15.74$ and $J-K=0.23$ is complex and formed of at least three components visible in the inset of Fig. 3. Two of them that are oriented approximately northsouth are relatively bright, whereas the one to the north-east corresponds to a faint diffuse feature. To the east of N88B lies a red bow-shaped filament with a curvature radius of $\sim 3^{\prime \prime}$ (Fig. 1) centered on N88B (see Sect. 3.6). This filament coincides with the narrow filament north-east of component B detected in the $\mathrm{H} \alpha$-band (HM99).

\subsection{JHK CM and CC diagrams}

Figure 8 shows a $K$ s vs. $J-K$ CM diagram of the N88 region. The magnitudes of the stars (Field 1) belonging to N88A are systematically underestimated (see Sect. 2.3). This difference is visible on the diagram where the stars of N88A (Field 2) 


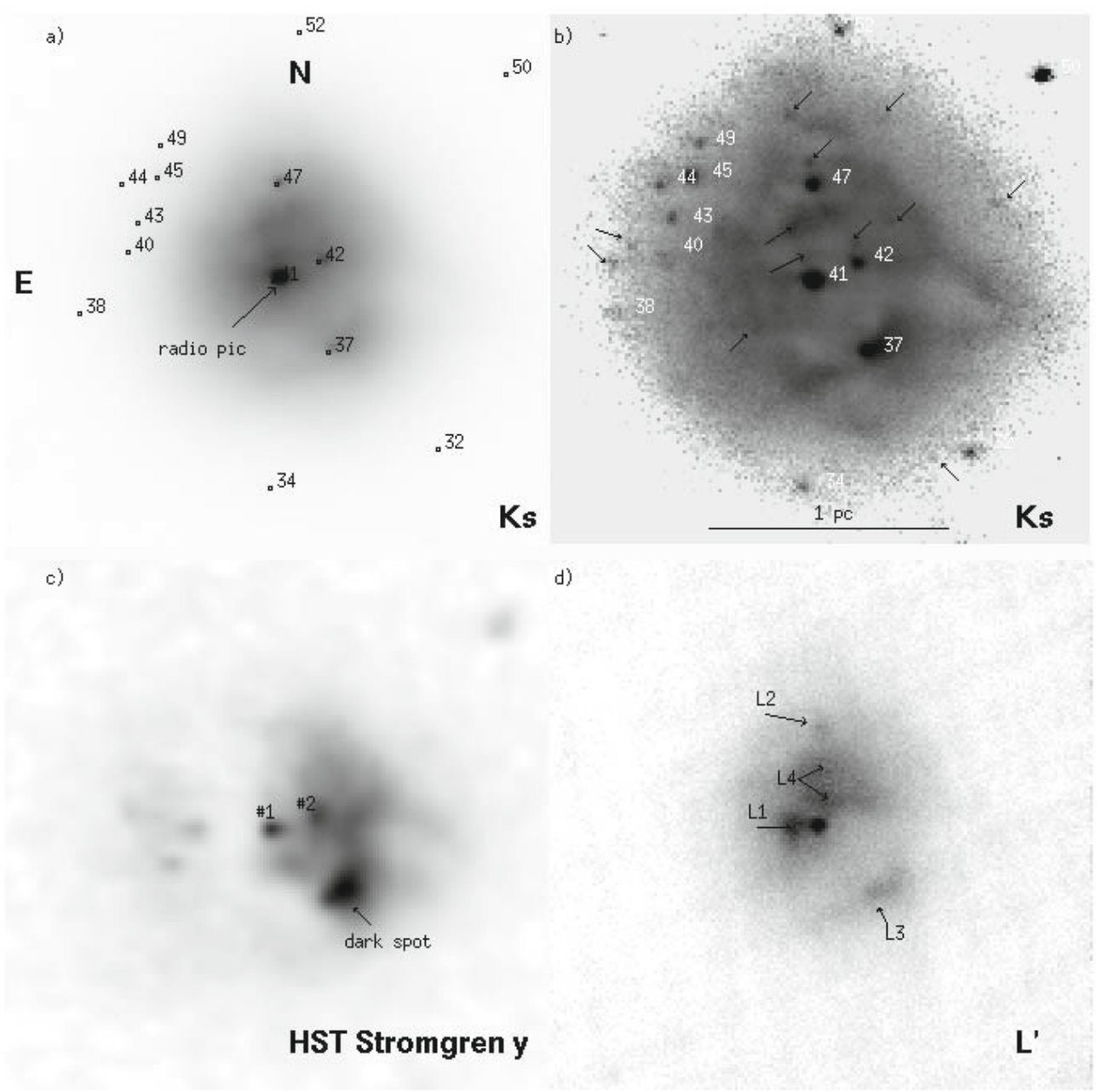

Fig. 4. Enlargements of the HEB N88A in Field 2. a) Ks-band obtained with the S27 camera. The numbering refers to Table 4 (Field 2). b) The DDP process applied to the $K$ s-band allows the enhancement of the faint stars of N88A-cl. A few stars not identified with DAOPHOT and small features are indicated by arrows. c) Stromgren $y$ image obtained with the HST showing the absorption lane, the stars \#1, \#2 as well as the "dark spot" of HM99. The stars \#1 and \#2 correspond to our stars \#41 and \#42. d) $L^{\prime}$-band obtained with the S27 camera, the different components are indicated. The field corresponds to $4.43^{\prime \prime} \times 4.32^{\prime \prime}$ (or $1.27 \mathrm{pc} \times 1.27 \mathrm{pc}$ ) and is outlined in Fig. 3 .

Table 4. Stars in N88A observed with the S27 camera (Field 2) in the Ks-band (numbers refer to Fig. 4).

\begin{tabular}{llllllll}
\hline \hline Id. & $\alpha(2000)$ & $\delta(2000)$ & $K^{\text {dao }}$ & err & $K^{\text {psf }}$ & $L^{\text {ap }}$ & $K-L$ \\
\hline 57 & 1248.88 & -73856.00 & 14.64 & 0.003 & & $14.36 \pm 0.05$ & \\
34 & 1247.94 & -7395.69 & 18.45 & 0.070 & & & \\
35 & 1247.64 & -7395.42 & 18.73 & 0.050 & & & \\
37 & 1247.83 & -7394.56 & 16.43 & 0.032 & $16.66 \pm 0.15$ & & \\
38 & 1248.30 & -7393.85 & 18.46 & 0.060 & & & \\
40 & 1248.21 & -7393.80 & 18.40 & 0.065 & & & \\
41 & 1247.93 & -7393.99 & 14.99 & 0.010 & $15.05 \pm 0.10$ & $14.1 \pm 0.10$ & 0.95 \\
42 & 1247.85 & -7393.82 & 16.11 & 0.047 & $16.60 \pm 0.30$ & & \\
43 & 1248.21 & -7393.30 & 17.62 & 0.066 & & & \\
44 & 1248.23 & -7393.05 & 17.76 & 0.060 & & & \\
45 & 1248.16 & -7393.14 & 17.12 & 0.044 & & & \\
47 & 1247.93 & -7393.19 & 15.62 & 0.036 & $15.99 \pm 0.20$ & & \\
49 & 1248.16 & -7392.70 & 17.41 & 0.061 & & & \\
51 & 1247.49 & -7392.29 & 18.27 & 0.040 & & & \\
52 & 1247.88 & -7391.90 & 18.65 & 0.061 & & & \\
L1-C & 1247.95 & -7393.80 & $\geq 18.5$ & 0.024 & & & \\
\hline
\end{tabular}

Notes. ${ }^{\text {dao }}$, psf and ap magnitudes derived using DAOPHOT, the PSF of star \#57, and an aperture respectively.

analyzed using the PSF of star\#57 (Table 4) are overplotted. However, taking into account the PSF given by DAOPHOT for
$J H K$ of about $0.2-0.3^{\prime \prime}$ we assume that the $J-K$ color values as well as $J-H$ and $H-K$ are correct within the uncertainties. 


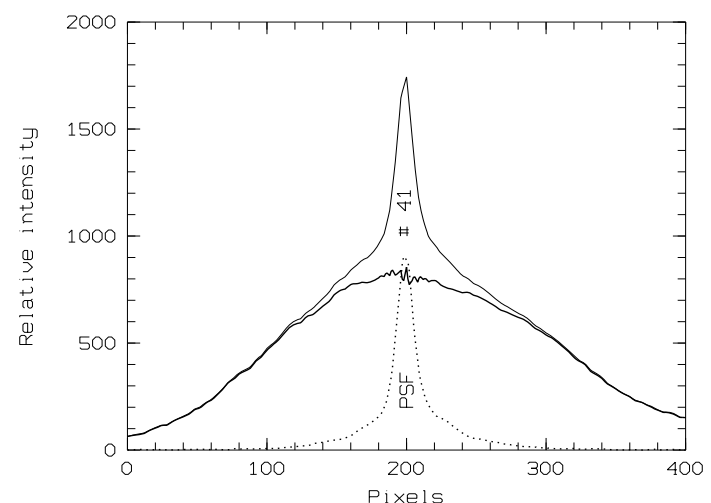

Fig. 5. The thin solid lines show the profile crossing the center of star \#41 without subtraction of the continuum. The dotted line shows the profile (PSF) of the isolated star \#40 labelled \#80 in Field 1. The intensity of the PSF of $F W H M=0.11^{\prime \prime}$ is multiplied by a normalization factor. The thick solid line shows the continuum obtained after the subtraction of the PSF. The plot range corresponds to 2.64".

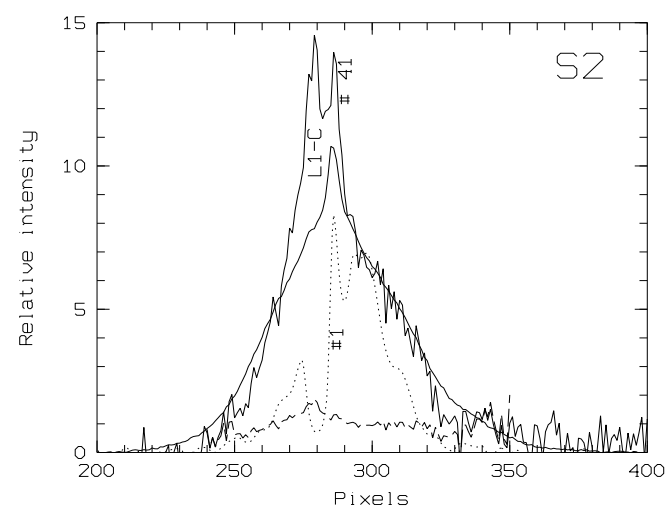

Fig. 6. East-west intensity distributions along the direction of slit $\mathrm{S} 2$ extracted from the $K$ s image (thin line), $L^{\prime}$ image (thick line) and Stromgren $y$ (HST) images (dotted line). The $L^{\prime} / K$ s ratio (dashed line) shows that the core L1-C (see Sect. 3.3.1) coincides with the minimum intensity of the absorption lane on the Stromgren $y$ plot. The plot range corresponds to $5.1^{\prime \prime}$.

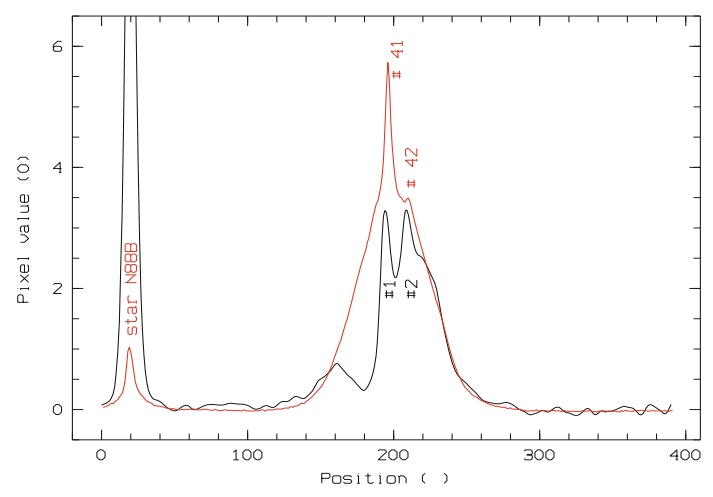

Fig. 7. Intensity distributions along the direction of slit S1 extracted from the $K$ s-band image (red line) and HST Stromgren $y$ images (black line). The Stars \#1, \#2 and the absorption lane (HM99) as well as our star \#41 and \#42 are indicated. The plot range corresponds to 10.6".

The color excess $E(B-V)$ towards N88 derived for hot stars from the Magellanic Clouds Photometric Survey (Zaritsky et al. 2002) in a radius of $1^{\prime}$ is small, $\sim 0.15$. In Fig. 8 the reddening track for $\mathrm{O}$ stars is plotted, assuming a total visual extinction $A_{\mathrm{v}}=5.8 E(J-K)$ (Tapia et al. 2003) and $A_{k}=0.112 A_{\mathrm{v}}$

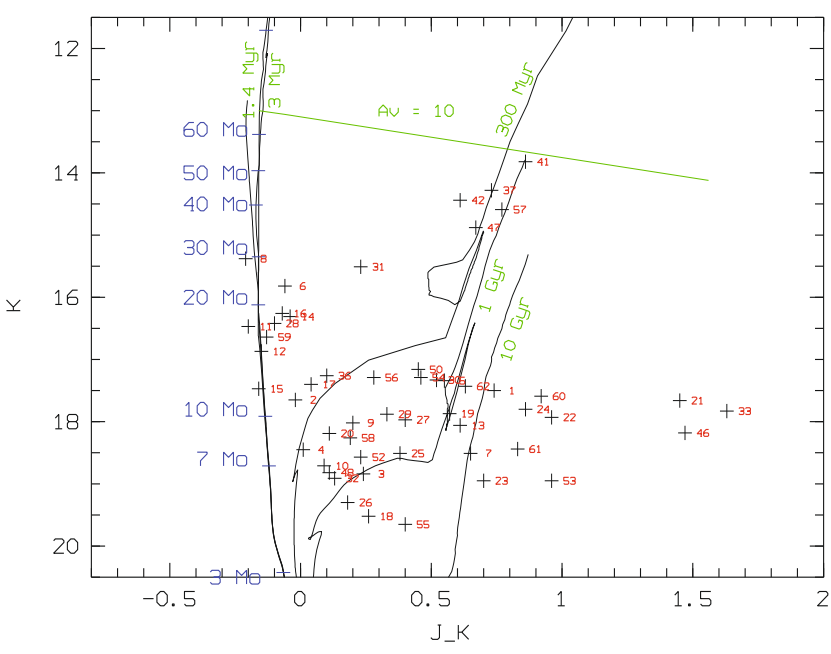

Fig. 8. $J-K \mathrm{CM}$ diagram for the point sources measured in N88 and N88A (Field 1). From left to right the $1.4 \mathrm{Myr}, 3 \mathrm{Myr}, 300 \mathrm{Myr}, 1$ and $10 \mathrm{Gyr}$ isochrones are overplotted (extinction free). Some masses between $3 M_{\odot}$ and $60 M_{\odot}$ are marked with a tick on the $3 \mathrm{Myr}$ isochrone. The stars \#37, \#41, \#42 and \#47 of Field 2 (Table 4) analyzed using NSTAR (crosses) and the PSF of star \#57 (filled squares) are overplotted.

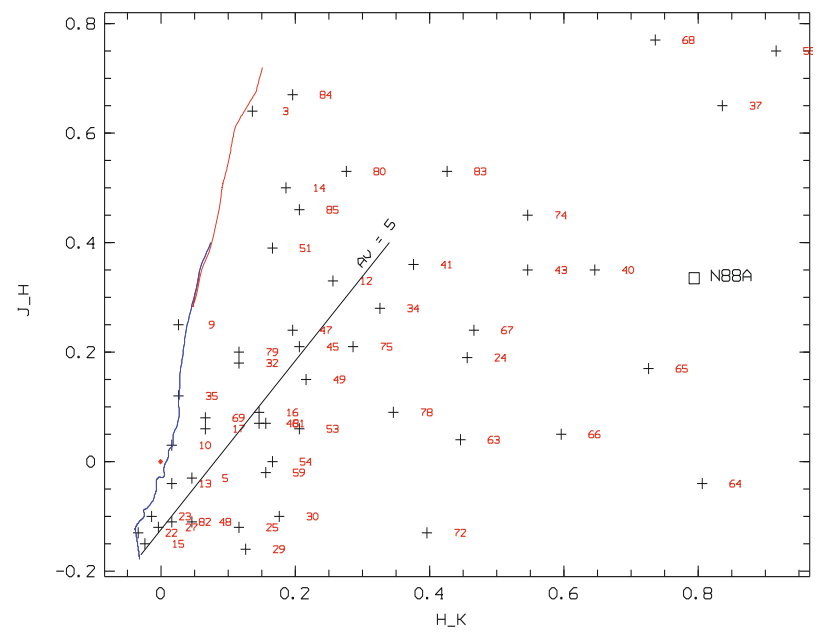

Fig. 9. CC diagram $(J-H$ vs. $H-K)$ for the measured stars in $\mathrm{N} 88$ and N88A. The blue line is the main sequence for stars of the age of $1 \mathrm{Myr}$ and the red line for stars of the age of $10 \mathrm{Myr}$. The empty squares represent the color-color of N88A integrated in an aperture of 4 " diameter and the filled squares the bright stars in N88A-cl. The solid line is the reddening vector up to $A_{\mathrm{v}}=5$.

(Rieke \& Lebosky 1985). It was derived using the star\#8 of type O9.5 in Wilcots 1994 b as reference. For this star we adopted a $(J-K)_{0}$ of $-0.15 \mathrm{mag}$ (Lejeune \& Shaerer 2001).

Several isochrones with different ages corresponding to $Z=$ 0.004 are overplotted (Fig. 8). The diagram appears to reveal two populations. The first one is a young population of dwarf and massive $\mathrm{O}$ stars, which appears to be fitted with the $3 \mathrm{Myr}$ isochrone. The second one could be a clump of red giant stars of $K$ magnitude in the range of 17-19.5 expanding in the age $300 \mathrm{Myr}-10 \mathrm{Gyr}$. The stars lying beyond the $10 \mathrm{Gyr}$ isochrones are likely to represent embedded stars situated deeper in the molecular cloud, young stars with circumstellar material or evolved stars surrounded by dust.

Figure 9 shows the $H-K$ vs. $J-H C C$ diagram. In this figure the solid line represents the reddening vector up to $A_{\mathrm{v}}=5$ mag. 
Table 5. Integrated NIR photometry of the whole region N88A.

\begin{tabular}{lcccccccc}
\hline \hline Reference & $J$ & $H$ & $K$ & $L^{\prime}$ & $J-K$ & $K \mathrm{~s}-L^{\prime}$ & $\begin{array}{c}F W H M \\
\left({ }^{\prime \prime}\right)\end{array}$ & $A_{\mathrm{v}}$ \\
& & & & & & & 4 & 7.3 \\
Denis $^{d}$ & 12.19 & & 11.11 & 1.08 & & 4 & 7.3 \\
2MASS $^{m}$ & 12.31 & 11.98 & 11.18 & 1.13 & & 4 & \\
Israel $^{i}$ & & & 11.05 & 8.92 & & 2 & 10 & 7.1 \\
this paper $^{2}$ & 12.15 & 11.92 & 11.08 & & & & 4 & 7 \\
\hline
\end{tabular}

Notes. ${ }^{(d)}$ Cioni et al. (2000); ${ }^{(m)}$ Cutri et al. $(2003) ;{ }^{(i)}$ Israel \& Koorneef (1991). From the Infrared Array Camera (IRAC) archive, Charmandaris et al. (2008) using an aperture of $\sim 7^{\prime \prime}$, give for the wavelength $3.6 \mu \mathrm{m}$ ( $L$-band), $4.5 \mu \mathrm{m}, 5.8 \mu \mathrm{m}$ and $8 \mu \mathrm{m}$ a magnitude for N88A of 9.52, 8.29, 7.1 and 5.58 mag respectively.

All stars that lie on the right side of the reddening vector should have IR-excess. Due to uncertainties on $H-K$ color we take into account only the stars beyond $\sim 0.1 \mathrm{mag}$ to the right of the reddening vector. Hence, the number of stars with IR-excess extracted from the CC diagram corresponds to at least $30 \%$ of the measured stars. On the CC diagram, obtained after integration in an aperture of $4^{\prime \prime}$ diameter (Table 5), N88A is overplotted and is found to the extreme right (Fig. 9). The plot shows a red $J-H$ color of 0.33 mag which contrasts with the blue $J-H$ color given by Israel \& Koorneef (1991).

\subsection{Search for YSO candidates in N88A and N88}

Due to their IR-excess emission, YSOs are positioned in the redder parts of the $\mathrm{CM}$ and $\mathrm{CC}$ diagrams. We first examined low spatial resolution $\left(\sim 2.5^{\prime \prime}\right)$ mid-IR Spitzer data of N88A (Charmandaris et al. 2008) and then the near-IR stellar content of both the regions N88A and N88 obtained with the high spatial resolution allowed by NACO $\left(\sim 0.10-0.3^{\prime \prime}\right)$. On the CM plot [3.6]-[8] versus [8], presented by Charmandaris et al. (2008), N88A lies at the border of the box representing the domain of Class II YSOs. N88A appears also inside the H II region domain. Similarly, on the CC [5.8]-[8] versus [3.6]-[8] diagram N88A is located near the $\mathrm{H}$ II region domain, but outside Class I and Class II YSO areas. These observations are explained by the fact that N88A is above all a very bright H II region with strong nebular emission lines and is affected by heavy extinction from local dust. In fact the Spitzer data represent flux integrations over the whole H II region $\left(\sim 1 \mathrm{pc}^{2}\right)$. Therefore, detecting a YSO inside the $\mathrm{H}$ II region seems hazardous unless the YSO is the dominant source inside N88A, which obviously is not the case. Note that although on the [5.8]-[8] versus [3.6]-[8] color diagram, based on model calculations (Whitney et al. 2004), N88A appears among Class 0 and Class I data points. This diagram is not applicable to the case of N88A for the reasons explained earlier.

In order to probe the presence of YSOs inside N88A we used our high resolution $J H K$ data. The stars \#37, \#41, \#42 and \#47 in N88A-cl are located at the upper part of the CM diagram (Fig. 8). In the $J H K$ CC diagram (Fig. 9), these stars exhibit an $H-K$ color ranging from 0.48 to $0.81 \mathrm{mag}$, and can be YSO candidates according to the JHK CC diagram of Maercker \& Burton (2005). However, their positions on the $J-K$ versus $K$ diagram (Fig. 8) suggest heavily reddened main-sequence (MS) massive stars of masses between $15 M_{\odot}$ and $30 M_{\odot}$. Their positions between the $300 \mathrm{Myr}$ and $1 \mathrm{Gyr}$ isochrones are also compatible

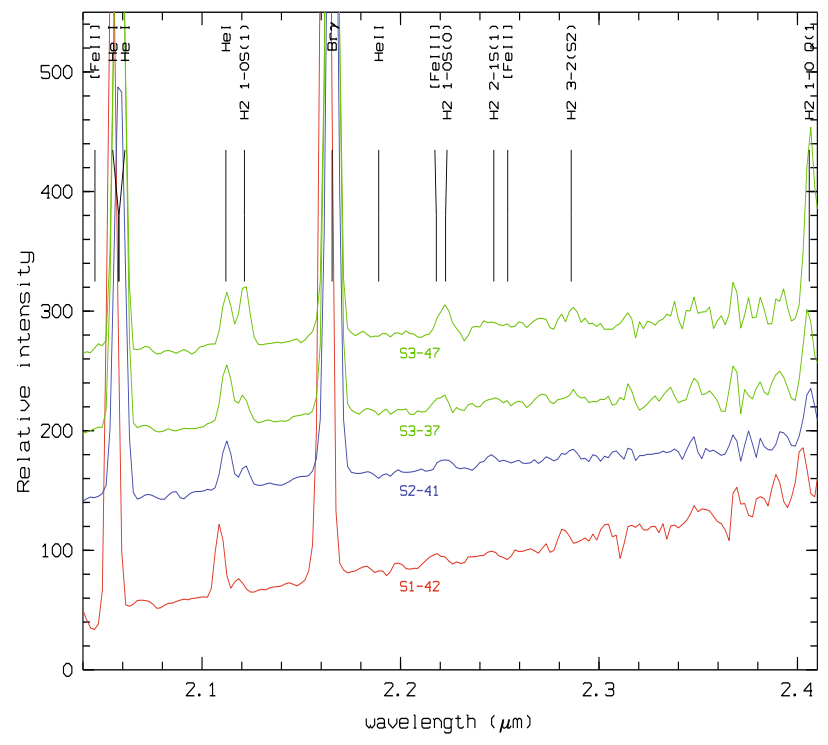

Fig. 10. One-dimensional spectra of the stars \#37, \#41, \#42 and \#47 plotted in the range of $2.04-2.41 \mu \mathrm{m}$.

with supergiants. If confirmed as supergiants, these stars would not be physically associated with N88A, which is very young. The assumption of reddened MS massive stars or massive YSOs seem more plausible. It is very difficult to distinguish between these possibilities. Consequently, caution must be applied, using only $J H K$ band observations to infer circumstellar material fraction in strong nebulous environments. The high spatial, but low spectral resolution S1-S3 spectra crossing the stars \#37, \#41, \#42 and \#47 (see Fig. 10) do not allow the accurate analysis of the $\mathrm{Br} \gamma$ line emission profile, which is characteristic for YSO sources, and new spectroscopic observations are needed to clarify the nature of these stars. In N88A, L1 presents a relatively bright peak (L1-C) in the $L^{\prime}$-band (Fig. 6). With $K-L^{\prime} \geq 4.5$, L1-C can be interpreted as a deeply embedded protostar (Lada et al. 2000). L1-C with a FWHM slightly larger than the PSF should still be in its contraction phase, surrounded by a dust shell. With a magnitude of 14 and a strong IR-excess (Table 4) L1-C could be a massive protostar of Class I.

We show from our high spatial resolution data of the $J H K$ CC diagram (Fig. 9) that in the extended N88 region at least $30 \%$ of the faint detected stars have an IR-excess. This takes into account the uncertainties which exclude some stars close to the reddening vector. These reddened stars seem to belong to a cluster of faint stars labeled N88cl (Fig. 1) and coincide with the young cluster HW81 (Hodge \& Wright 1977), which is formed of bright stars, situated towards N88 and not affected by dust (HM99). The JHK CC diagram (Fig. 9) shows that the bright stars \#6, \#8, \#11, \#12, \#15, \#28 and \#59 in HW81 have no IR-excess, and their masses spread in the range of $15 M_{\odot}$ to $30 M_{\odot}$. In N88-cl most of the reddened stars have a mass $\leq 12 M_{\odot}$ (Fig. 8) and are probably intermediate-mass YSO candidates. We assume that N88-cl belongs to N88 (Fig. 1). The stars \#21, \#33 and \#46 of N88-cl aligned on the east filament of N88B (Fig. 1) with an $H-K$ excess $>0.7 \mathrm{mag}$, could be good YSO candidates (Fig. 9). Their alignment suggests that their formation may be triggered by the expansion of the shell around N88B. 
A\&A 510, A95 (2010)

Table 6. Relative integrated HeI, HI and $\mathrm{H}_{2}$ emission-line fluxes in N88A for 14 positions extracted across S1-S3 (Fig. 12abc).

\begin{tabular}{llllllll}
\hline \hline $\mathrm{Id}$ & $\mathrm{HeI}$ & $\mathrm{HeI}$ & $\mathrm{H}_{2} 1-0 \mathrm{~S}(1)$ & $\mathrm{Br} \gamma$ & $\mathrm{H}_{2} 1-0 \mathrm{~S}(0)$ & $\mathrm{H}_{2} 2-1 \mathrm{~S}(1)$ & $I(\mathrm{He} 2.113 \mu \mathrm{m}) / I(\mathrm{Br} \gamma)$ \\
& $2.058 \mu \mathrm{m}$ & $2.113 \mu \mathrm{m}$ & $2.121 \mu \mathrm{m}$ & $2.166 \mu \mathrm{m}$ & $2.223 \mu \mathrm{m}$ & $2.247 \mu \mathrm{m}$ & \\
\hline $\mathrm{S} 1-41$ & 3.18 & 0.375 & 0.121 & 6.07 & 0.099 & 0.031 & 0.062 \\
$\mathrm{~S} 1-42$ & 3.36 & 0.337 & 0.075 & 5.94 & 0.092 & 0.044 & 0.057 \\
S2-41 & 2.38 & 0.257 & 0.094 & 4.76 & 0.068 & 0.038 & 0.054 \\
S3-37 & 0.76 & 0.065 & 0.028 & 1.21 & 0.018 & 0.009 & 0.054 \\
S3-47 & 0.60 & 0.055 & 0.066 & 1.01 & 0.033 & 0.006 & 0.054 \\
S1-1 & 2.43 & 0.24 & 0.199 & 4.30 & 0.126 & 0.069 & 0.054 \\
S1-2 & 0.17 & 0.017 & 0.094 & 0.31 & 0.054 & 0.035 & 0.057 \\
S2-1 & 1.49 & 0.16 & 0.150 & 3.24 & 0.093 & 0.048 & 0.049 \\
S3-1 & 0.67 & 0.065 & 0.049 & 1.11 & 0.033 & 0.009 & 0.059 \\
S3-2 & 0.58 & 0.057 & 0.026 & 0.95 & 0.018 & 0.009 & 0.060 \\
S3-3 & 0.02 & 0.002 & 0.017 & 0.04 & 0.010 & 0.008 & 0.056 \\
S3-4 & 0.02 & & 0.019 & 0.02 & 0.009 & 0.009 & 0.065 \\
S3-p1 & 0.74 & 0.092 & 0.020 & 1.42 & 0.014 & 0.009 & 0.058 \\
S3-p2 & 0.89 & 0.090 & 0.036 & 1.55 & 0.034 & 0.008 & \\
\hline
\end{tabular}

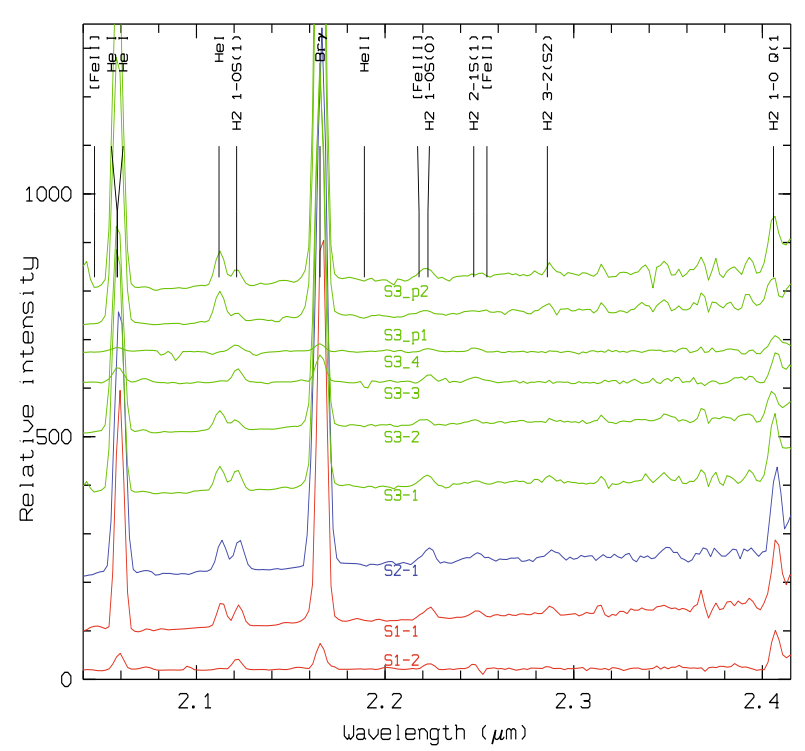

Fig. 11. One-dimensional nebular spectra plotted in the range of $2.04-2.41 \mu \mathrm{m}$.

\subsection{The ionizing sources}

\subsubsection{N88A}

At $3 \mathrm{~cm}$ radio emission, Indebetouw et al. (2004) found for N88A a Lyman continuum flux of $\log N_{\mathrm{Lyc}}=49.5$. Using the spectral classification of Smith et al. (2002) we estimate from this flux the type of the ionizing source of N88A to range from $\mathrm{O} 4$ to $\mathrm{O} 5$. The type of the ionizing source derived by HM99 using the $\mathrm{H} \beta$ flux corresponds to an O6 star. The extracted 1D spectra at different positions along the slits $\mathrm{S} 1-\mathrm{S} 3$ crossing N88A (Fig. 3) are listed in Table 6. In each position the rows are averaged and the corresponding 1D spectra are shown in Fig. 10 for the stars and Fig. 11 for nebular emission. The positions of these spectra are represented by horizontal line segments on the plots corresponding to the distribution of the $\mathrm{Br} \gamma$, He I 2.058, 2.113 and $\mathrm{H}_{2} 2.121 \mu \mathrm{m}$ emission lines as well as the continuum emission (Figs. 12a-c). The length of the segment is proportional to the number of lines integrated along the slit. All the emission lines are continuum subtracted. This figure also indicates the $\mathrm{H}_{2}$ components $\mathrm{C} 1-\mathrm{C} 3$ (see Sect. 3.5) of which the distribution intends to clarify the presence of the structures seen
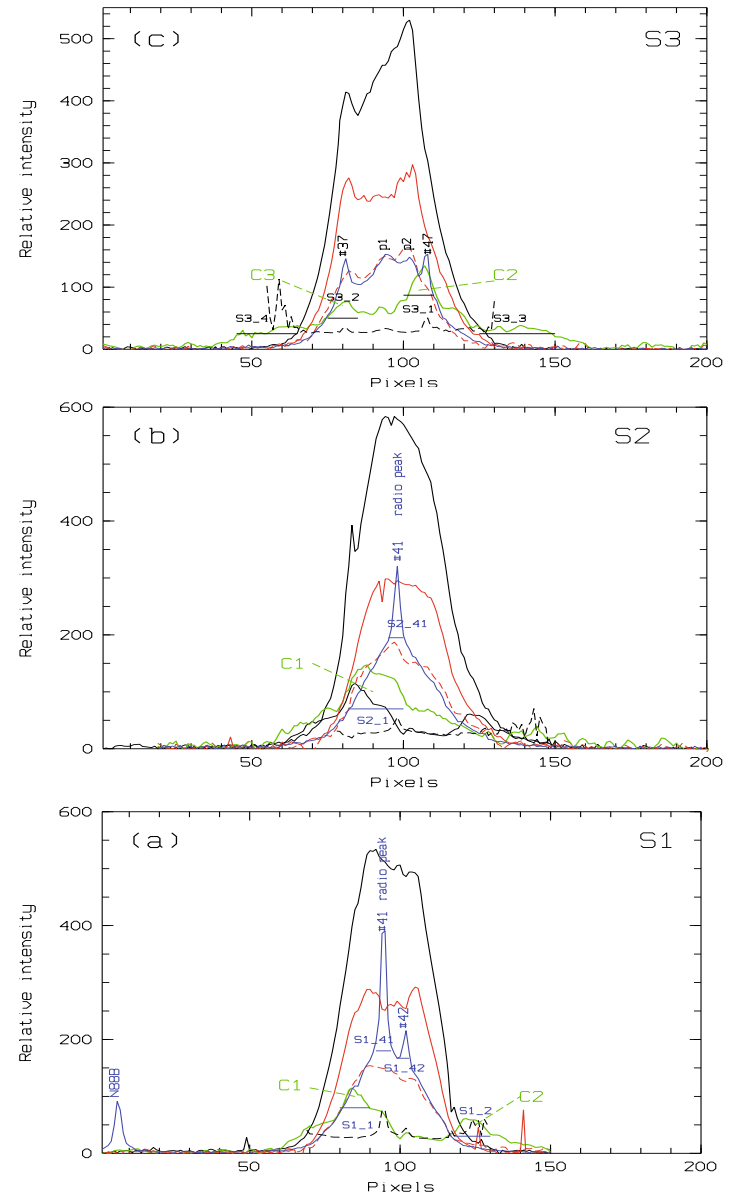

Fig. 12. a) Relative intensity distributions along slit $\mathrm{S} 1$ aligned on stars \#31, \#41 and \#42 (Fig. 2). b) Relative intensity distributions along slit S2 crossing the star \#41. c) Relative intensity distributions along slit S3 aligned on stars \#37 and \#47. The red line represents the intensity distribution of the He I $2.058 \mu \mathrm{m}$ emission line. The dashed red line represents the He I $2.113 \mu \mathrm{m}$ and the green one the $\mathrm{H}_{2} 2.121 \mu \mathrm{m}$, both are multiplied by a factor five. The continuum/Br $\gamma$ ratio multiplied by a factor 100 is plotted (dashed line). The position of each 1D spectrum is indicated by a small horizontal segment (solid line). The plot range corresponds to $10.6^{\prime \prime}\left(1 \mathrm{pix}=0.05273^{\prime \prime}\right)$.

in Fig. 13 (see Sects. 3.6 and 3.7). From the spectra presented in Figs. 10 and 11 we derive a ratio $\mathrm{He}$ I $2.113 \mu / \mathrm{Br} \gamma$ lines 
of the mean value of 0.06 which indicates a hot $\mathrm{O}$ star of $T_{\text {eff }} \geq 40000 \mathrm{~K}$ (Hanson et al. 2002). Table 6 (Col. 8) shows that this ratio is fairly constant across N88A.

In the spectra of the stars \#37, \#41, \#42 and \#47 (Fig. 10) the He II $2.185 \mu \mathrm{m}$ absorption is not detected, if present. The detection is difficult because of our low signal/noise and our low spectral resolution. The NIII $2.115 \mu \mathrm{m}$ is not detected either. When the He II $2.185 \mu \mathrm{m}$ absorption line is not present (Bik et al. 2005), the spectral type of a star should be later than O8 V, which is the case for our four resolved stars. As seen above, the spectral type of the ionizing source of the whole nebula is derived from radio and $\mathrm{H} \beta$ flux and ranges between $\mathrm{O} 4$ and O6. We will adopt a type $\mathrm{O} 5$ for our computation. Its comparison with the type $08 \mathrm{~V}$ derived from our spectroscopy for the bright central star \#2-41 clearly shows that other massive stars must contribute to the ionization of N88A. The flux excess between the ionizing star \#41 of type $\mathrm{O} 8 \mathrm{~V}$ and the $\mathrm{O} 5$ type derived from the flux could be produced by at least five $\mathrm{O} 8 \mathrm{~V}$ stars. The massive stars \#37, \#42, and \#47 (Fig. 4) located in the upper part of the CM diagram could be good candidates for the ionization of N88A. The $3 \mathrm{~cm}$ radio peak centered at $(\alpha, \delta)=$ $\left(1^{\mathrm{h}} 24^{\mathrm{m}} 7^{\mathrm{s}} .9,-73^{\circ} 9^{\prime} 4^{\prime \prime}\right)$, and our images show that the radio peak coincides perfectly with the central bright star \#41 $\left(1^{\mathrm{h}} 24^{\mathrm{m}} 7^{\mathrm{s}} .95\right.$, $-73^{\circ} 9^{\prime} 3^{\prime \prime} 8$ ) (Table 4). This strong radio emission superposing the NIR emission Br $\gamma$ line $2.16 \mu \mathrm{m}$ line (Figs. 12a and b) is characteristic for an $\mathrm{H}$ II region.

\subsubsection{The N88A-cl cluster}

On the $J H K$ image of Lada et al. (2000) the Trapezium region of the size of $\sim 0.75 \times 0.75 \mathrm{pc}$, which is located at a distance of $450 \mathrm{pc}$, contains four bright central massive stars and a plethora of low-mass stars with IR-excess. In our $K$ s-band N88A has an approximately similar diameter (Fig. 4b) and also contains the cluster N88A-cl. This cluster contains other resolved stars not identified by DAOPHOT as well as unresolved stars in crowded groups (Fig. 4b). Among these stars the four brightest ones analyzed using the $J H K$ bands also exhibit IR-excess. N88A with its cluster appears morphologically comparable with the Trapezium region and other compact star formation regions of similar size, like SH2 269 in our Galaxy, and N159-5 in the LMC (Testor et al. 2007). N88A-cl can also be compared with the pre-main-sequence (PMS) clusters, candidate YSOs, of size $0.24 \mathrm{pc}$ to $2.4 \mathrm{pc}$ found in SMC-N66 (Gouliermis et al. 2008). Like N88A-cl these PMS clusters are found to coincide with [OIII], $\mathrm{H} \alpha$ and $\mathrm{H}_{2}$ emission peaks (see Sect. 3.7). Their clustering properties are similar to the star forming region Orion despite its higher metallicity (Hennekemper et al. 2008).

\subsubsection{The bright central star \#41}

In the Trapezium, the four ionizing bright stars lie within a diameter of $\sim 0.05 \mathrm{pc}$. At the distance of the SMC our spatial resolution is $\sim 0.03 \mathrm{pc}(\sim 6900 \mathrm{AU})$. With this comparatively lower spatial resolution it cannot be excluded that the ionizing star \#41 could also be a tight young cluster. This assumption is strengthened by the photometry. The magnitude of this star obtained using the PSF is 15.05 (Table 4). Its dereddened magnitude derived with $A_{K}=0.58 \mathrm{mag}$ corresponds to a mass of $\sim 40 M_{\odot}$ (Fig. 8). Using the parameters for O stars of Vacca (1996), we classify \#41 as a O6.5 V type star instead of O8 V derived by spectrocopy, and it could also be complex.

\subsection{The continuum dust emission}

Through the $J H K$-band, N88A presents a relatively bright nebular continuum emission centered on star \#41 (Figs. 4a and 5). In the $L^{\prime}$-band the continuum is less homogeneous, due to the brightness of L1-L4. Although the signal to noise ratio is not high, L4 appears very faint in the $y$-band image (Fig. 4c). The nature of this continuum is not clear. The relatively strong $L$-band excess of $K-L^{\prime}=2$ mag derived from the integration of the whole region N88A (Table 4) supposes that the continuum could come from the emission of circumstellar material (Lada et al. 2000) around resolved and unresolved young stars. These stars could be located mainly at the positions L1-L4 (Fig. 4d). L4 shows two peaks P1 and P2 visible on the continuum plot of S3 (Fig. 12c). But the continuum emission could also be formed by interstellar dust associated with the gas of the CO cloud (Testor et al. 1985, HM99; Stanamirovic et al. 2000; Israel et al. 2003). In Fig. 4c the strong optical emission at the position of the "dark spot" could be explained by strong dust scattering that reflects the light of at least star \#37. The nature of the continuum emission of N88A should be a combination of the two possibilities: emission of circumstellar material and/or dust associated with the gas. Along the slits S1-S3 the intensity distribution of the continuum near the $\mathrm{Br} \gamma$ line shows a strong continuum/Br $\gamma$ ratio of $0.30-0.4$ over a range of $6^{\prime \prime}$ around star \#41 (Figs. 12a,b). In these figures the broadness of the continuum and $\mathrm{Br} \gamma$ distribution are similar $\left(F W H M \sim 1.8^{\prime \prime}\right)$.

\section{7. $\mathrm{H}_{2}$ emission}

In Testor et al. (2005) the profile of the $\mathrm{H}_{2}$ emission along the slit corresponding to our spectrum S1 appears in the form of two blended profiles, due to the low spatial resolution spectroscopy. Thanks to the high spatial resolution of our new data, the complex morphology of the $\mathrm{H}_{2}$ emission in N88A is revealed both by imagery and spectroscopy. Figure 13 shows a bidimensional image of the $\mathrm{H}_{2}$ emission ( $v=1-0 \mathrm{~S}(1)$ line). This image is achieved by subtracting the image in the $2.24 \mu \mathrm{m}$ filter, which allows the only passage of the continuum radiation from the image in the $2.13 \mu \mathrm{m}$ filter. In this $\mathrm{H}_{2}$ image, N88A resembles a circular shell (a) of a diameter of $\sim 3^{\prime \prime}$ with three maxima labelled $\mathrm{C} 1-\mathrm{C} 3$ (Fig. 13). Within (a) there is a cavity that suggests radiation pressure, especially from the four central stars. The structure $\mathrm{C} 2$ is very bright and extended along the northeastsouthwest direction and coincides with the ionization front detected by HM99. C2 has a sharp extension in the direction of star \#47. The $\mathrm{H}_{2}$-band image also shows that the bow-shaped filament located east of N88A seems to belong to a second shell (b) of a diameter of 7" centered on N88B (Fig. 13). The shells (a) and (b) seem to be in interaction approximately at the position $\mathrm{C} 1$.

Unlike the spectra obtained with ISAAC (Testor et al. 2005), the high-spatial resolution long-slit spectra $\mathrm{S} 1-\mathrm{S} 3$ allow the resolution of the inner structures and stars of N88A. In the direction of the slit $\mathrm{S} 1$, the two $\mathrm{H}_{2}$ emission structures $\mathrm{C} 1$ and $\mathrm{C} 2$ are well resolved (Fig. 12a) and separated by $\sim 2^{\prime \prime} . \mathrm{C} 1$ distant of $\sim 0.5^{\prime \prime}$ from \#41 coincides with the absorption lane observed in optical images by Kurt et al. (1999) and HM99. In the direction of the slit S2, only the structure $\mathrm{C} 1$ is seen east of star \#41 (Fig. 12b). In the direction of the slit S3, the well seen structures C2 and C3 (Fig. 12c) coincide with the stars \#47 and \#37 respectively. According to Rubio et al. (2000), massive star formation could be taking place in dense $\mathrm{H}_{2}$ knots associated with molecular clumps. According to Gouliermis et al. (2008) 


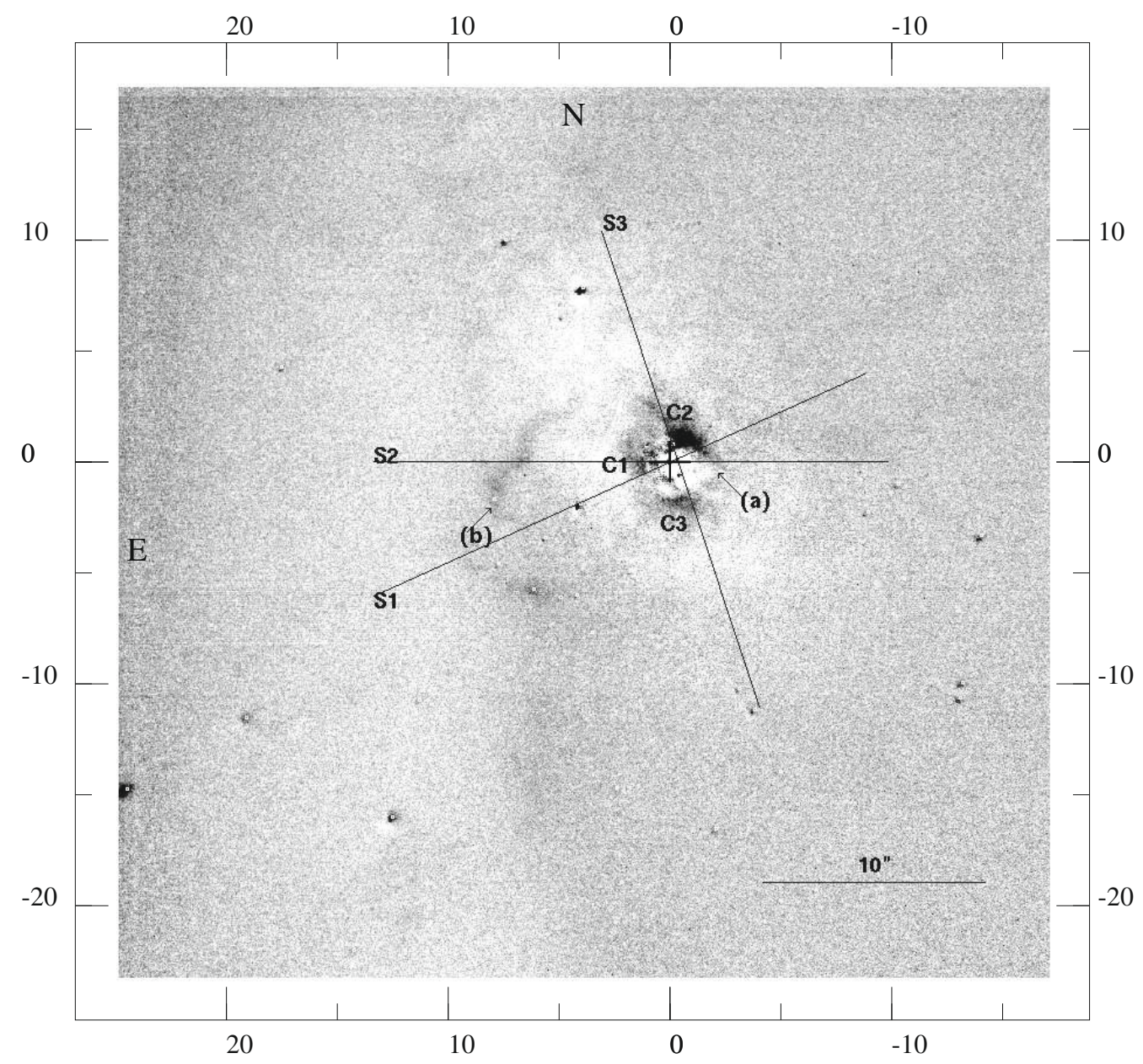

Fig. 13. The $\mathrm{H}_{2} 2.121 \mu \mathrm{m}$ emission (continuum subtracted) showing the three components $\mathrm{C} 1-\mathrm{C} 3$ of the shell a) as well as the larger shell b). The $\mathrm{RA}$ and Dec offsets $(\operatorname{arcsec})$ are from star \#41, represented by a cross (coordinates 0,0 ). The image size is $42^{\prime \prime} \times 40^{\prime \prime}$, corresponding to $\sim 13 \times 12 \mathrm{pc}$.

PMS clusters could be candidate YSOs. These results strengthen our assumption that N88A-cl could contain YSOs.

From Israel \& Koorneef (1988), the molecular hydrogen emission may be caused either by shock excitation due to embedded stars or by fluorescence of molecular material in the ultraviolet radiation field of the OB stars exciting the $\mathrm{H}$ II region in the molecular cloud. They conclude that in the MCs, shock excitation of $\mathrm{H}_{2}$ is only expected very close to (i.e. $0.15 \mathrm{pc}$ ) stars embedded in a molecular cloud. At a larger distance, radiative excitation of $\mathrm{H}_{2}$ by the UV radiation field of the OB stars is the only mechanism. Their spectrophotometry with a large aperture $\left(10^{\prime \prime}\right)$ made a precise determination of the brightness of the lines $2-1(\mathrm{~S} 1), 1-0(\mathrm{~S} 1)$ and $1-0(\mathrm{~S} 0)$ difficult. This is usually considered between shocks and radiative excitation. These lines, deblended when necessary, have been derived from our lowspectral-resolution spectra $\mathrm{S} 1-\mathrm{S} 3$, crossing the zones $\mathrm{C} 1-\mathrm{C} 3$ as well as the stars \#41, \#42, \#37 and \#47. Their intensities are shown in Table 6. None of these lines suffer from atmospheric absorption, considering a $V_{\mathrm{lsr}}$ of $147 \mathrm{~km} \mathrm{~s}^{-1}$ (Israel et al. 2003), as it can be derived from the solar spectrum atlas (Livingston \& Wallace 1991) with the help of a useful home-made software $^{2}$. The lines may suffer from differential reddening. Mathis (1990) estimates that the effect for Galactic Sources follows a power-law in the $J$-, $H$-, and $K$-bands: $I_{1} / I_{2}=\left(\lambda_{1} / \lambda_{2}\right)^{-1.7}$. The

\footnotetext{
2 http://www.u-cergy. fr/LERMA-LAMAP/informatique/ raiesH2/index.html
}

effect on the $v=1-0 \mathrm{~S}(0)$ and $v=2-1 \mathrm{~S}(1)$ lines would be that they are overestimated by $10 \%$. However, this is based entirely on Galactic Sources. To the best of our knowledge, a differential reddening law has not been determined for Extra-Galactic Sources. Moreover, since the effect is already within our observational uncertainty, we choose to ignore it. In Fig. 14 the strengths of the $2.247 \mu \mathrm{m} \mathrm{2-1(S1)}$ and $2.223 \mu \mathrm{m} \mathrm{1-0(S1)} \mathrm{lines}$ are shown relative to the $2.121 \mu \mathrm{m} 1-0(\mathrm{~S} 1)$ line for all the objects in Table 6. For radiative excitation (PDRs), the usual criteria are that these ratios should range from 0.5 to 0.6 and from 0.4 to 0.7 respectively (Black \& van Dishoeck 1987), while for shock-excitation with $T=2000 \mathrm{~K}$ they should be 0.08 and 0.21 respectively (Shull \& Hollenbach 1978). We show in Fig. 14 the results from Draine \& Bertoldi (1996) reported by Hanson et al. (2002) for high density $\left(n_{\mathrm{H}}=10^{6}\right.$ and $\chi=10^{4}$ and $\left.10^{5}\right)$ and low density $\left(n_{\mathrm{H}}=10^{4}\right.$ and $\left.\chi=10^{2}\right)$ PDRs. We also show in this figure results for more recent and elaborated PDR models (Le Petit et al. 2006) (for $n_{\mathrm{H}}$ ranging from $10^{4}$ to $10^{7}$ and $\chi$ from $10^{3}$ to $10^{7}$ ) as well as for shock models (Flower \& Pineau des Forêts 2003) (for the same range of $n_{\mathrm{H}}, v_{\mathrm{S}}$ from 10 to $60 \mathrm{~km} \mathrm{~s}^{-1}$, the magnetic scaling factor $b$ from 0 to 10 and an ortho/para ratio of 3). None of these models, either PDRs or shocks really fit with our observations, with the exception of the objects S3-p1 and p2 as well as S3-1 that are well inside the nebulosity and could fit with shocks (relatively high velocity $v_{\mathrm{S}}, 30$ to $50 \mathrm{~km} \mathrm{~s}^{-1}$ ) and low $b \sim 0.1$. Nevertheless, apart from the three $\mathrm{H}_{2}$ lines mentioned above, four additional ones are observed: $2-1 \mathrm{~S}(2), 2-1 \mathrm{~S}(3)$, 


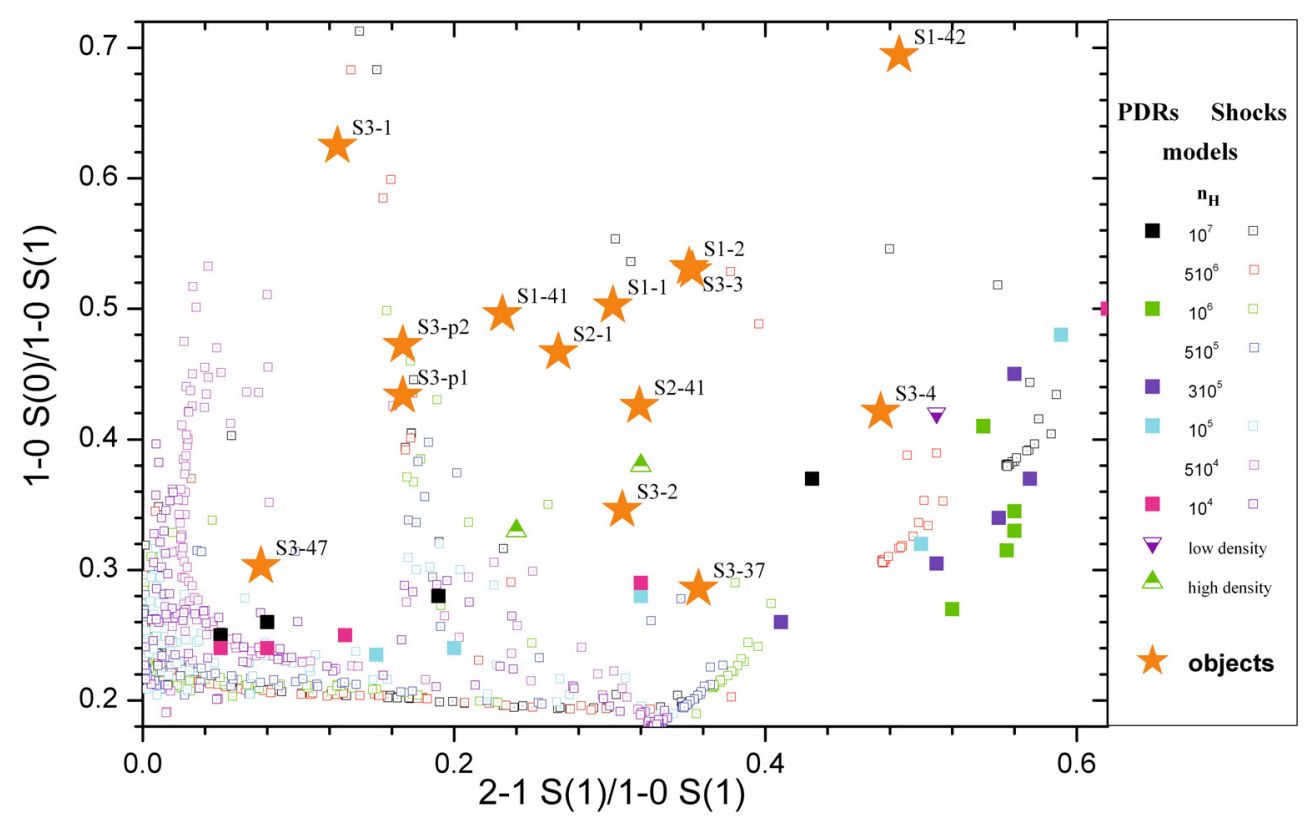

Fig. 14. 2-1 $S(1) / 1-0(\mathrm{~S} 1)$ vs. $1-0 \mathrm{~S}(0) / 1-0 \mathrm{~S}(1)$ ratio at different positions in the HEB N88A.

3-2 S(1) and especially 3-2 $\mathrm{S}(2)$ at respectively $2.154,2.073$, 2.386 and $2.286 \mu \mathrm{m}$. These lines are more sensitive to absorption by atmospheric lines and depend in fact on the accuracy of the $v_{\text {lsr }}$. The first one is the least affected by positive or negative velocity shift, whilst the two others are slightly absorbed up to $180 \mathrm{~km} \mathrm{~s}^{-1}$ but may suffer a $50 \%$ absorption at $140 \mathrm{~km} \mathrm{~s}^{-1}$. The last one is free of absorption between 115 and $150 \mathrm{~km} \mathrm{~s}^{-1}$ and does not appear to be blended with any other lines. Possible turbulence in the emitting region may first broaden the lines, then lower the effect due to atmospheric absorption. In any case the concomitant appearance of lines emanating from high $v$ or $J$ as the 3-2 $\mathrm{S}(2) \mathrm{H}_{2}$ line shows a clear trend in favor of the major presence of PDR excitation for most of the observed objects, but without totally excluding the additional presence of shock excitation. Clearly both higher spatial and spectral resolutions are required to make progress in the knowledge of these objects.

\section{Conclusions}

We present high spatial resolution imaging of $F W H M \sim$ $0.12^{\prime \prime}-0.25^{\prime \prime}$ in the $J H K L^{\prime}$-band of the HEB N88A and its immediate environment, and the main results are as follows:

N88A is associated with a cluster that contains at least thirteen stars centered approximately on the bright central star \#41, which could be complex.

N88A coincides perfectly with the $3 \mathrm{~cm}$ radio peak and is probably ionized not only by the star \#41 classified of type $\mathrm{O} 8 \mathrm{~V}$, but also by other low to high-mass stars.

From the analysis of the JHK CC diagram we found four possible MYSO star candidates in the N88A cluster, and also three probable YSOs in the red bow east of N88A. In N88 at least $30 \%$ of the detected stars have an IR-excess.

From the $K-L$ excess we found that the core L1-C in N88A is probably a heavily embedded, high mass protostar of Class I.

The continuum emission at the position of \#41 is very bright and represents about $30 \%$ of the Br $\gamma$ emission peak.

The $\mathrm{H}_{2}$ infrared emission in N88A resembles a shell formed mainly by three peaks, one of which coincides with the ionization front.
We show that the excitation mechanism may be caused predominantly by PDRs, without excluding combination with shocks.

The morphology of N88A could be comparable with galactic regions such as the nearby Trapezium region in the Orion nebula.

Future $J H K$ band imaging data, using higher spatial resolution and longer wavelengths like $L^{\prime}$ and $M^{\prime}$ provided by the NACO S13 camera are still needed to disentangle the IR-excess origin in N88A. Higher spectral resolution spectra are also required to obtain a better analysis of the different spectral lines like the $\mathrm{Br} \gamma$ emission. These new observations should then allow us to investigate the HEB N88A more thoroughly. This object, which is the brightest, the most excited and reddened of the MCs, presents a unique opportunity to make progress in the knowledge of newborn massive stars in regions of low metallicity.

Acknowledgements. We would like to thank the Directors and Staff of the ESOVLT for making these observations possible and particularly the NACO team for their excellent support. J.L.L., L.K. and S.D. would like to acknowledge the support of the French PCMI program "Physico Chimie du Milieu Interstellaire", funded by the CNRS. This research has made use of the Simbad database, VizieR and Aladin operated at CDS, Strasbourg, France, and the NASA's Astrophysics Data System Abstract Service.

\section{References}

Black, J. H., \& van Dishoeck, E. F. 1987, ApJ, 322, 412

Bik, A., Kaper, L., Hanson, M. M., \& Smits, M. 2005, A\&A, 440, 121

Bik, A., Kaper, L., \& Waters, L. B. F. M. 2006, A\&A, 455, 561

Cesaroni, R., Galli, D., Lodato, G., Walmsley, C. M., \& Zhang, Q. 2007, in Protostars and Planets V, ed. B. Reipurth, D. Jewitt, \& K. Keil, 197

Charmandaris, V., Heydari-Malayeri, M., \& Chatzopoulos, E. 2008, A\&A, 487, 567

Chen, C. H. R., Chu, Y.-H., Gruendl, R. A., Gordon, K. D., \& Heisch, F. 2009, ApJ, 695, 511

Cioni, M.-R., Loup, C., Habing, H. J., et al. 2000, A\&AS, 144, 235

Cole, A. 1998, ApJ, 500, L137

Cutri, R. M., et al. 2003, The IRSA 2MASS All-Sky Point Source Catalog, NASA/IPAC Infrared Science Archive, http://irsa.ipac.caltech. edu/applications/Gator/

Draine, B. T., \& Bertoldi, F. 1996, ApJ, 468, 269D

Flower, D. R., \& Pineau des Forêts, G. 2003, MNRAS, 343, 390

Gouliermis, D., Chu, Y.-H., Henning, T., Brandner, W., \& Gruendl, R. 2008, ApJ, 688, 1050 
Hanson, M., Luhman, K., \& Rieke, G. H. 2002, ApJS, 138, 35

Henize, K. G. 1956, ApJS, 2, 315

Hennekemper, E., Gouliermis, D., Henning, T., Brandner, W., \& Dolphin, A. 2008, ApJ, 672, 914

Heydari-Malayeri, M., \& Testor, G. 1982, A\&A, 111, L11

Heydari-Malayeri, M., Le Bertre, T., \& Magain, P. 1988, A\&A, 195, 230

Heydari-Malayeri, M., Charmandris, V., Deharveng, L., Rosa, M. R., \& Zinnecker, H. 1999, A\&A, 347, 841

Heydari-Malayeri, M., Rosa, M. R., Charmandris, V., et al. 2007 [arXiv : 0707.1209H]

Hodge, P. W., \& Wright, F. W. 1977, The Small Magellanic Cloud (Seattle: University of Washington Press)

Indebetouw, R., Johnson, K., \& Conti, P. 2004, AJ, 128, 2206

Israel, F. P., \& Koorneef, J. 1988, A\&A, 190, 21

Israel, F. P., \& Koorneef, J. 1991, A\&A, 248, 404

Israel, F. P., Johansson, L., Rubio, M., et al. 2003, A\&A, 406, 817

Kovacs, G. 2000, A\&A, 360, L1

Kurt, C. M., Dufour, R. J., Garnett, D. R., et al. 1999, ApJ, 518, 246

Lada, C. J., Muench, A. A., Haisch, K. E., Jr., et al. 2000, ApJ, 120, 3162

Livingston, W., \& Wallace, L. 1991, NSO Technical Report, Tucson: National Solar Observatory, National Optical Astronomy Observatory

Lejeune, T., \& Shaerer, D. 2001, A\&A, 366, 538

Le Petit, F., Nehmé, C., Le Bourlot, J., \& Roueff, E. 2006, ApJS, 164, 506

Mathis, J. 1990, ARA\&A, 28, 37

Maercker, M., \& Burton, G. 2005, A\&A, 438, 663

Martin-Hernandez, N. L., Peeters, E., \& Tielens, A. G. G. M. 2008, A\&A, 489 1189

McGee, R. X., \& Newton, L. M. 1982, Proc. Astron. Soc. Aust., 4, 308

McNamara, D. H., \& Feltz, K. A. 1980, PASP, 92, 587
Pugliese, G., Christou, J., Koehler, R., \& Drummond, J. 2002, AAS, 201, 5505

Rieke, G., \& Lebofsky, M. 1985, ApJ, 288, 618

Rubio, M., Contursi, A., Lequeux, J., et al. 2000, A\&A, 359, 1139

Schmeja, S., Gouliermis, D., \& Klessen, R. 2009, ApJ, 694, 367

Shull, J. M., \& Hollenbach, D. J. 1978, ApJ, 220, 525

Simon, J. D., Bollato, A., Stanimirovic, S., et al. 2008, in The 2nd Annual Spitzer Science Center Conf.: Infrared Diagnostics of Galaxy Evolution, ASP Conf. Ser. 381 [arXiv: astro-ph/0603834v1]

Smith, L. J., Noriss, R.P. F., \& Crowther, P. A. 2002, MNRAS, 337, 1309

Stanimirovic, S., Staveley-Smith, L., van der Hulst, J. M., et al. 2000, MNRAS, 315,791

Stanghellini, L., Villaver, E., Shaw, R., \& Mutchler, M. 2003, ApJ, 598, 1000

Stetson, P. B. 1987, PASP, 99, 191

Tapia, M., Persi, P., Roth, M., et al. 2003, MNRAS, 339, 44

Testor, G. 2001, A\&A, 372, 667

Testor, G., \& Pakull, M. 1985, A\&A, 145, 170

Testor, G., Lemaire, J. L., \& Field, D. 2003, A\&A, 407, 905

Testor, G., Lemaire, J. L., Field, D., \& Callejo, G. 2005, A\&A, 434, 497

Testor, G., Lemaire, J. L., Field, D., Kristensen, L. E., \& Diana, S. 2007, A\&A, 469,459

Vacca, W. D., Garmany, C. D., \& Shull, J. M. 1996, ApJ, 460, 914

Westerlund, B. E. 1997, The Magellanic Clouds (Cambridge: Cambridge Univ.Press), 28

Whitney, B. A., Indebetouw, R., Bjorkman, J. E., \& Wood, K. 2004, ApJ, 617, 1177

Wilcots, E. M. 1994b, AJ, 108, 1674

Zaritsky, D., Harris, J., Thompson, I., Grebel, E. K., \& Massey, P. 2002, AJ, 123, 855 